\title{
Flammability conditions for ultra-lean hydrogen premixed combustion based on flame-ball analyses
}

\author{
Eduardo Fernández-Tarrazo , Antonio L. Sánchez , Amable Liñán , \\ Forman A. Williams
}

\begin{abstract}
A B S T R A C T
It has been reasoned that the structures of strongly cellular flames in very lean mixtures approach an array of flame balls, each burning as if it were isolated, thereby indicating a connection between the critical conditions required for existence of steady flame balls and those necessary for occurrence of self-sustained premixed combustion. This is the starting assumption of the present study, in which structures of near-limit steady sphericosymmetrical flame balls are investigated with the objective of providing analytic expressions for critical combustion conditions in ultra-lean hydrogen-oxygen mixtures diluted with $\mathrm{N}_{2}$ and water vapor. If attention were restricted to planar premixed flames, then the lean-limit mole fraction of $\mathrm{H}_{2}$ would be found to be roughly ten percent, more than twice the observed flammability limits, thereby emphasizing the relevance of the flame-ball phenomena. Numerical integrations using detailed models for chemistry and radiation show that a onestep chemical-kinetic reduced mechanism based on steady-state assumptions for all chemical intermediates, together with a simple, optically thin approximation for water-vapor radiation, can be used to compute near-limit fuel-lean flame balls with excellent accuracy. The previously developed one-step reaction rate includes a crossover temperature that determines in the first approximation a chemical-kinetic lean limit below which combustion cannot occur, with critical conditions achieved when the diffusion-controlled radiation-free peak temperature, computed with account taken of hydrogen Soret diffusion, is equal to the crossover temperature. First-order corrections are found by activation-energy asymptotics in a solution that involves a near-field radiation-free zone surrounding a spherical flame sheet, together with a far-field radiation-conduction balance for the temperature profile. Different scalings are found depending on whether or not the surrounding atmosphere contains water vapor, leading to different analytic expressions for the critical conditions for flame-ball existence, which give results in very good agreement with those obtained by detailed numerical computations. The one-step chemistry employed in the present work, which involves a non-Arrhenius rate having a cutoff at the crossover temperature, applies with excellent accuracy to the description of lean premixed hydrogen-air combustion, i.e, for $\phi \leq 0.5$ at atmospheric pressure, and could be used for instance in the numerical simulation of the propagation of curved or cellular flames in ultra-lean reactive atmospheres, of interest for safety analyses related to the storage, transport, and handling of hydrogen.
\end{abstract}




\section{Introduction}

Although discussions of the utilization of hydrogen for zeroemission vehicles usually revolve around fuel cells, ultralean combustion in reciprocating engines is potentially viable for power production at least as cleanly. In designing such engines, accurate knowledge of the lean flammability limits is essential. Moreover, especially if exhaust-gas recirculation is to be considered, those limits in the presence of water vapor must be known. Such knowledge is relevant, for example, to proposed laser-induced spark ignition [1]. Because of the interest in this application, as well as safety concerns in explosion prevention, the study reported here addresses ultra-lean hydrogen flammability limits at different pressures, temperatures, and water-vapor content, complementing existing experimental results $[2,3,4,5]$.

Diffusive-thermal instabilities are known to influence deflagration propagation in very lean hydrogen-air mixtures [6]. Combustion is enhanced by effects of preferential diffusion, which produce superadiabatic flame temperatures and enable cellular flames to propagate in mixtures with hydrogen content well below the flammability limit computed theoretically for steady planar deflagrations. For sufficiently lean mixtures, the cellular flame breaks up into separate cells, which propagate as an array, leaving behind a finite amount of unburnt fuel. In near-limit situations, the cells close upon themselves to form flame balls with a nearly spherical shape [7]. Interactions between neighboring flame balls become weak because of the large separating distance. Under those conditions, the flame can be envisioned as an ensemble of isolated flame balls, which propagates with a velocity that can be determined by adding the individual flame-ball burning rates, with an additional assumption introduced for the average inter-flame-ball spacing [8]. This limiting mode of cellular-flame propagation clearly fails when the conditions are too lean to support self-sustained flame-ball combustion. For this reason, analyses of structures of isolated flame balls can provide limiting flammability conditions for fuel-lean mixtures of hydrogen and oxygen that may or may not include inert species such as nitrogen.

The existence of nonpropagating steady spherical flame balls was first predicted theoretically by Zeldovich [9] and verified experimentally years later under microgravity conditions $[10,11,12,13]$. As suggested by Zeldovich [9] (see, also [14]), radiative heat losses stabilize these otherwise unstable diffusion-reaction structures. The resulting branch of stable solutions, corresponding to relatively large flame-ball radii, on the order of $1 \mathrm{~cm}$, has been investigated theoretically with a one-step Arrhenius reaction $[15,16]$. Numerical studies, including detailed-chemistry mechanisms and different models for radiation $[17,18,19]$, have shown that radiative heat losses are very significant, keeping the peak temperature of hydrogen-air flame balls below $1200 \mathrm{~K}$ along the whole branch of stable solutions. As emphasized recently [20], under those near-crossover conditions, all chemical intermediates appear in very small concentrations and obey chemical-kinetic steady-state approximations. Consequently, a reduced chemical-kinetic mechanism involving a single global reaction $2 \mathrm{H}_{2}+\mathrm{O}_{2} \rightarrow 2 \mathrm{H}_{2} \mathrm{O}$, previously derived for planar deflagrations near the lean flammability limit $[21,22]$, can be employed to describe hydrogen-air flame balls with excellent accuracy. The corresponding overall rate, given below in [11], involves two factors with a strong temperature dependence, so that it can be approximated close to the flammability limit by

$\omega \propto \exp \left(\mu \beta \frac{T-\mathrm{T}_{\mathrm{c}}}{\mathrm{T}}\right)\left[\exp \left(\beta \frac{\mathrm{T}-\mathrm{T}_{\mathrm{c}}}{\mathrm{T}}\right)-1\right] \mathrm{Y}_{\mathrm{H}_{2}}^{2}$,

proportional to the square of the hydrogen mass fraction $Y_{\mathrm{H}_{2}}$ and involving two activation energies $\beta \simeq 10$ and $\mu \beta \simeq 7$ along with a crossover temperature $T_{c}$ such that $\omega=0$ for $T=T_{c}$, with a zero rate $\omega=0$ replacing Eq. (1) for $T<T_{c}$. The value of $T_{c}$ is determined from the condition, given below in Eq. (17), that the rate of the branching reaction $\mathrm{H}+\mathrm{O}_{2} \stackrel{1}{\rightleftharpoons} \mathrm{OH}+\mathrm{O}$ be equal to effective rate of $\mathrm{H}$-atom recombination, proportional to the rate of $\mathrm{H}+\mathrm{O}_{2}+\mathrm{M} \stackrel{4 f}{\rightarrow} \mathrm{HO}_{2}+\mathrm{M}$ and involving a composition-dependent factor $\alpha$. Because of the very high $\mathrm{H}_{2} \mathrm{O}$ chaperon efficiency of this last reaction, the resulting value of $T_{c}$ varies with the amount of water vapor, which in turn depends on the composition of the fresh mixture.

The one-step chemistry, involving the non-Arrhenius rate displayed above, applies with excellent accuracy to the description of lean premixed hydrogen-air combustion, i.e, for values of the equivalence ratio $\phi \leq 0.5$ at atmospheric pressure, where

$\phi=\frac{8 \mathrm{Y}_{\mathrm{H}_{2 \omega}}}{\mathrm{Y}_{\mathrm{O}_{2 \infty}}}$

when expressed as a function of the ambient mass fractions of hydrogen and oxygen. This one-step chemistry could be used in numerical and theoretical investigations of flame stability as well as dynamics of cellular fronts, thereby facilitating the numerical investigation of the intermediate range of flame structures encountered for decreasing values of the equivalence ratio, from steady planar flames to highly cellular flames involving an ensemble of flame balls [8]. An example of such applications include the direct numerical simulation of propagating flame cells, which becomes computationally expensive when detailed chemistry is used [23]. Also, numerical simulations of three-dimensional flame-ball dynamics, previously attempted with a generic Arrhenius chemistry model [24,25], could make use of the one-step $\mathrm{H}_{2}-\mathrm{O}_{2}$ reduced mechanism for a more realistic description, enabling the flame-ball response to be linked directly to the ambient conditions.

It was seen in [20] that, because of the large value of $\beta$ present in the one-step rate, at leading order the flame-ball size is determined by the condition that radiation removes enough of the chemical heat for the flame temperature to remain close to the crossover value. The leading-order analytic solution computed in this way describes with excellent accuracy the variation of the flame-ball radius $r_{f}$ with equivalence ratio $\phi$ along the upper branch of stable solutions, except near the lean limit, where it fails to give the turning point of the $r_{f}-\phi$ curve. The corresponding near-turningpoint analysis, providing the flammability limit below which no flame ball may exist, is derived in the present work.

The paper begins by using numerical integrations to guide the selection of the initial conservation equations for the 
analytical development, including simplified models for the chemistry and radiation. The radiation-free solution is investigated next to determine the leading-order solution for the lean flammability limit as well as the characteristic scales involved in the problem. Activation-energy asymptotics is then employed to find an analytical solution near the turning point, which requires scalings that are different depending on whether the ambient mixture does or does not contain water vapor. A two-term analytic expressions is then obtained for the critical hydrogen content at the turning point. The theoretical predictions are tested by comparisons with results of numerical integrations for atmospheric hydrogen-air flame balls with detailed chemistry and radiation models, showing excellent agreement for various extents of dilution with water vapor and nitrogen.

\section{Simplifications to the chemistry and radiation descriptions}

The structure of steady, spherically symmetrical flame balls is determined by integration of the convection-free energy and species conservation equations written for the temperature $T$ and mass fractions $Y_{i}$ of species $i$, including detailed descriptions for the transport, chemistry, and radiation, with symmetry conditions at the center and ambient values $T=T_{\infty}$ and $Y_{i}=Y_{i \infty}$ in the far field $[17,18,19]$. In fuel-lean hydrogenair flame balls, the molecular transport is dominated by the abundant presence of nitrogen and oxygen, so that the thermal conductivity and the molecular diffusivities of the different species can be computed with the simple temperature-dependent laws suggested in [26] for methaneair flames. As shown previously [20], Soret diffusion of light species needs to be included if accurate results are desired. Besides, the computation requires specification of the chemical-reaction scheme, an appropriate choice being the so-called San Diego Mechanism of 21 steps [27,28]. It also requires taking appropriate account of $\mathrm{H}_{2} \mathrm{O}$ radiation, with different levels of complexity being employed in previous studies. The most elaborate radiation model includes both emission and absorption, with radiative transport computed by a Statistical Narrow Band (SNB)- Discrete Ordinate method $[29,30]$, while the simplest model assumes an optically thin medium, an approximation used, for instance, in $[17,18,19]$. We shall see below that the latter description becomes increasingly accurate as the flame-ball radius decreases near the lean limit, and it can be used, in particular, to compute the critical composition at the turning point with reasonably small errors that, in fact, are entirely negligible when water vapor is absent in the ambient mixture. As explained by Ronney [30], larger errors emerge for mixtures diluted with $\mathrm{CO}_{2}$; for such cases, not considered here, an optically thin model is never a good approximation.

The results of the integrations for flame balls including detailed chemistry and an SNB model for radiation are shown in Figs. 1 and 2. The plots exhibit the variation with equivalence ratio of the flame-ball radius $r_{f}$, defined as the location where the rate of hydrogen consumption reaches a maximum, along with the variation of the peak temperature $\mathrm{T}_{\max }$, which is always reached close to $r=r_{f}$. The

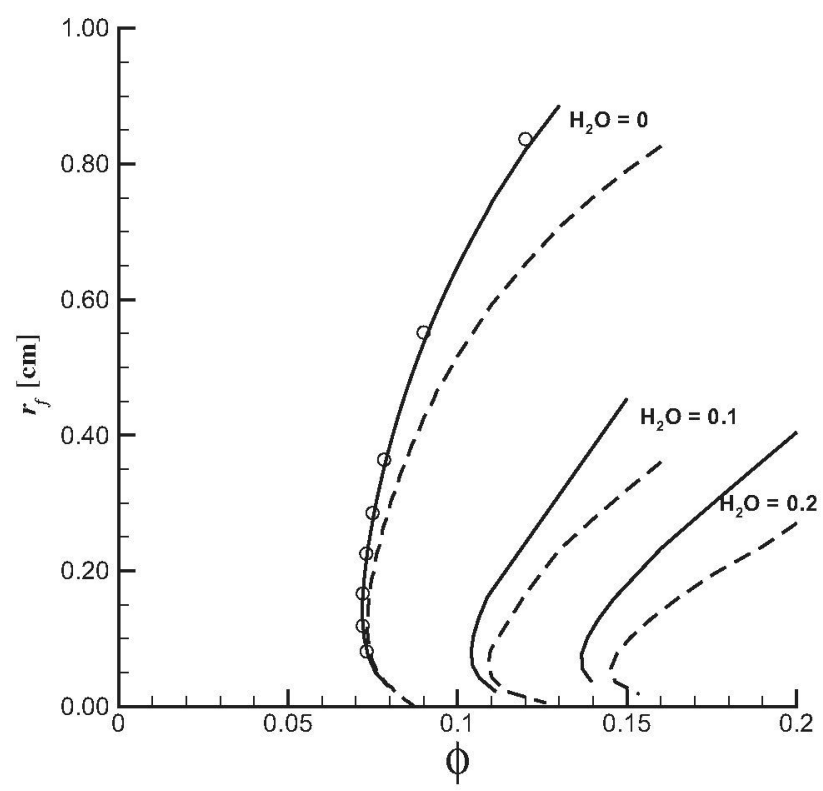

Fig. 1 - The variation with the equivalence ratio $\phi$ of the flame-ball radius as obtained from numerical integrations with the SNB radiation model and the 21-step chemical scheme (solid curves), with the SNB radiation model and the one-step reduced mechanism (circles), and with the optically thin approximation given in Eq. (18) and the onestep reduced mechanism (dashed curves); for three different values of the mass fraction of water vapor in the ambient atmosphere.

computations are for an ambient hydrogen-air mixture at pressure $p=1$ atm and normal ambient temperature $\mathrm{T}_{\infty}=300 \mathrm{~K}$, and they also include test cases with water-vapor dilution corresponding to ambient mass fractions $\mathrm{Y}_{\mathrm{H}_{2} \mathrm{O}_{\infty}}=0.1$ and $\mathrm{Y}_{\mathrm{H}_{2} \mathrm{O}_{c o}}=0.2$. As expected, two different solutions are found for values of $\phi$ above a critical value $\phi_{l}$ and no solution exists for $\phi<\phi_{1}$.

Along with the plots of peak temperature, the computations for dry air in Fig. 2 include the variation with composition of the crossover temperature, as defined from Eq. (17). As can be seen, the resulting peak temperature is not too far above $T_{c}$, with values $T_{\max }-T_{c} \sim \beta^{-1} T_{c}$. Under such lowtemperature conditions, the previously derived one-step reduced mechanism becomes applicable [21,22], and it is seen in the comparisons shown in Figs. 1 and 2 that results for $\mathrm{H}_{2}$-air flame balls computed with the reduced chemistry, represented by circles, are very accurate. The one-step formula can therefore be used with confidence as a replacement for the 21-step chemistry to facilitate the analysis.

Simplifications to the SNB description of radiative heat losses are also needed to enable analytical results to be developed. Assuming the characteristic absorption length to be much larger than the characteristic flame-ball size leads to the well-known optically thin approximation, used in previous studies $[17,18,19,20]$. The results obtained with the one-step approximation for the chemistry combined with the optically thin approximation for radiation are shown as dashed curves in Figs. 1 and 2. As can be seen, in the range of $\phi$ 


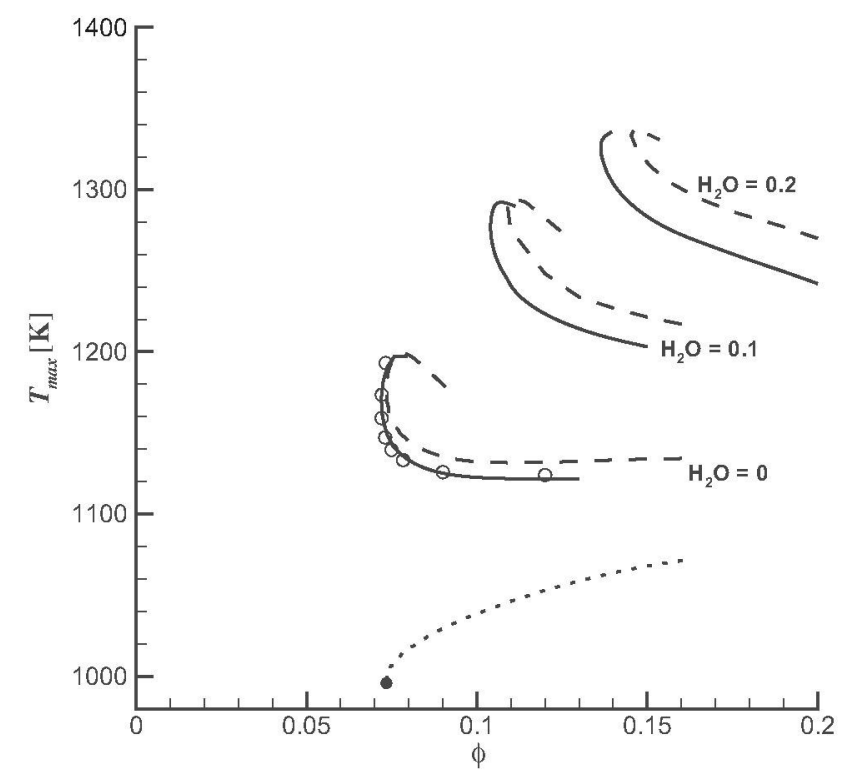

Fig. 2 - The variation with the equivalence ratio $\phi$ of the peak temperature as obtained from numerical integrations with the SNB radiation model and the 21-step chemical scheme (solid curves), with the SNB radiation model and the one-step reduced mechanism (circles), and with the optically thin approximation given in Eq. (18) and the onestep reduced mechanism (dashed curves); for three different values of the mass fraction of water vapor in the ambient atmosphere. The dotted curve represent the variation of the crossover temperature $T_{c}$, at which the rate (11) of the one-step computation with optically-thin radiation vanishes for dry air, with the solid circle representing the values obtained at the turning point.

considered, the optically thin approximation yields moderate errors in flame-ball radii, on the order of $20 \%$, in agreement with previous numerical investigations [30]. Note, however, that the accuracy is much better for the computation of flammability conditions. In particular, for the dry hydrogenair mixture, the turning point obtained with the optically thin approximation is extremely close to that determined with the SNB model. More noticeable departures appear with water vapor in the atmosphere, leading to overpredictions of $\phi_{1}$ on the order of $5 \%$. Since these errors are comparable to those expected from the asymptotic analysis presented below, the simple radiation law associated with the optically thin approximation, given below in Eq. (18), will be adopted in the following development.

Before proceeding with computations of steady flame balls it is worth commenting briefly on their stability. The solutions along the branch of small flame-ball radii, with almost negligible radiation, are known to be unstable to one-dimensional disturbances $[15,16]$, whereas radiation helps stabilize the solutions along the upper branch in Fig. 1 (i.e., the lower branch in Fig. 2), which correspond to the large flame balls found in microgravity experiments $[10,11,12,13]$. Previous numerical computations [19] and theoretical analyses [16] indicate that the critical solution at the turning point is unstable, with the first stable solution appearing in the upper branch for an equivalence ratio slightly larger than $\phi_{l}$. This stability characteristic was further verified in the present work by performing a number of transient computations, with the steady solution used as initial condition. The results indicated, in particular, that the instability for the solution along the upper branch occurs as a subcritical Hopf bifurcation. For the computations with a dry ambient shown in the figure, the bifurcation occurs for $\phi \simeq 0.0753$. This value is in fact very close to the turning-point value $\phi_{1}=0.0735$, so that, for practical purposes, one may in principle neglect unsteady effects in defining the critical conditions for flame-ball existence. For this reason, in the following the flammability limit will be associated with the turning point of the steady solution.

Although flame-ball stability and dynamics will no longer be considered here, it should be noted that the scalings and flame-ball asymptotic structure identified below could be employed in future one-dimensional and three-dimensional stability analyses, the latter affecting the solution when the mixture becomes sufficiently rich $[15,16]$. The scalings also could be employed in bifurcation studies of self-drifting flame balls $[31,32]$. Many further investigations thus could be based on the formulation identified in the present paper.

\section{Problem formulation}

In the one-step approximation, only three chemical species $\left(\mathrm{H}_{2}, \mathrm{O}_{2}\right.$ and $\left.\mathrm{H}_{2} \mathrm{O}\right)$ need to be considered, so that the solution for the spherically symmetric, steady flame ball is determined by integrating

$$
\frac{1}{r^{2}} \frac{\mathrm{d}}{\mathrm{d} r}\left(\rho \mathrm{D}_{\mathrm{O}_{2}} r^{2} \frac{\mathrm{d} Y_{\mathrm{O}_{2}}}{\mathrm{~d} r}\right)=-\mathrm{W}_{\mathrm{O}_{2}} \omega
$$

$\frac{1}{r^{2}} \frac{\mathrm{d}}{\mathrm{d} r}\left(\rho D_{\mathrm{H}_{2} \mathrm{O}} r^{2} \frac{\mathrm{d} Y_{\mathrm{H}_{2} \mathrm{O}}}{\mathrm{d} r}\right)=2 \mathrm{~W}_{\mathrm{H}_{2} \mathrm{O}} \omega$

$-\frac{1}{r^{2}} \frac{\mathrm{d}}{\mathrm{d} r}\left(\rho \mathrm{D} r^{2} \frac{\mathrm{d} Y}{\mathrm{~d} r}\right)=-2 \mathrm{~W}_{\mathrm{H}_{2}} \omega$

and

$-\frac{1}{r^{2}} \frac{\mathrm{d}}{\mathrm{d} r}\left(\lambda r^{2} \frac{\mathrm{dT}}{\mathrm{d} r}\right)=-\mathrm{Q}_{\mathrm{R}}+2 \mathrm{~W}_{\mathrm{H}_{2}} q \omega$,

with boundary conditions $\mathrm{dT} / \mathrm{d} r=\mathrm{d} \mathrm{Y}_{\mathrm{i}} / \mathrm{d} r=0$ at $r=0$ and $\mathrm{T}-\mathrm{T}_{\infty}=\mathrm{Y}-\mathrm{Y}_{\mathrm{H}_{2 \infty}}=\mathrm{Y}_{\mathrm{O}_{2}}-\mathrm{Y}_{\mathrm{O}_{2 \infty}}=\mathrm{Y}_{\mathrm{H}_{2} \mathrm{O}}-\mathrm{Y}_{\mathrm{H}_{2} \mathrm{O}_{\infty}}=0$ as $r \rightarrow \infty$. Here, $r$ is the distance from the flame-ball center, $\rho$ is the density of the gas mixture, and $W_{i}$ is the molecular weight of species $i$.

As previously explained, in fuel-lean hydrogen-air flame balls the thermal conductivity $\lambda$ is dominated by nitrogen and oxygen, so that the approximate expression

$\lambda / c_{p}=2.58 \times 10^{-5}(\mathrm{~T} / 298)^{0.7} \mathrm{~kg} /(\mathrm{ms})$,

suggested in [26] for methane-air flames, also dominated by these species, can be used with excellent accuracy. The specific heat at constant pressure $c_{p}=\sum_{i} c_{p i} Y_{i}$ is evaluated with use made of NASA polynomial fits for the temperature 
dependence of $c_{p i}$ for each species. At the same level of approximation [26], the molecular diffusivity $D_{i}$ can be computed as the binary diffusion coefficient of the species $i$ into nitrogen, according to

$\rho D_{i}=2.58 \times 10^{-5}(\mathrm{~T} / 298)^{0.7} / \mathrm{L}_{\mathrm{i}}, \mathrm{kg} /(\mathrm{ms})$,

with Lewis numbers given by $L_{i}=(1.11,0.30,0.83)$ for $\left(\mathrm{O}_{2}, \mathrm{H}_{2}, \mathrm{H}_{2} \mathrm{O}\right)$, respectively. Following [20], to incorporate in Eq. (5) the thermal diffusion and the molecular diffusion in a single Fickian-like diffusion equation the formulation introduces a modified fuel mas fraction

$\mathrm{Y}=\left(\mathrm{T} / \mathrm{T}_{\infty}\right)^{\alpha_{\mathrm{H}_{2}}} \mathrm{Y}_{\mathrm{H}_{2}}$,

and a modified diffusivity

$D=\left(T / T_{\infty}\right)^{-\alpha_{\mathrm{H}_{2}}} D_{\mathrm{H}_{2}}$,

with $\alpha_{\mathrm{H}_{2}}=-0.29$ representing the $\mathrm{H}_{2}$ Soret factor [33]. The rate $\omega$ (moles per unit volume per unit time) for the overall reaction $2 \mathrm{H}_{2}+\mathrm{O}_{2} \rightarrow 2 \mathrm{H}_{2} \mathrm{O}$ is given by [21]

$\omega=\frac{1}{G H}\left(\frac{k_{1 j}}{\alpha k_{4 j} C_{M}}-1\right) \frac{k_{2 f} k_{3 f}}{k_{1 b}}\left(\rho Y_{\mathrm{H}_{2}} / \mathrm{W}_{\mathrm{H}_{2}}\right)^{2}$,

where the subscripts $f$ and $b$ denote the forward and backward specific reaction-rate constants for the steps $\mathrm{H}+\mathrm{O} \stackrel{1}{\rightleftharpoons} \mathrm{OH}+\mathrm{O}$, $\mathrm{H}_{2}+\mathrm{O} \stackrel{2}{\rightleftharpoons} \mathrm{OH}+\mathrm{H}, \mathrm{H}_{2}+\mathrm{OH} \stackrel{\stackrel{3}{\rightleftharpoons}}{\rightleftharpoons} \mathrm{H}_{2} \mathrm{O}+\mathrm{H}$, and $\mathrm{H}+\mathrm{O}_{2}+\mathrm{M} \stackrel{4 f}{\rightarrow} \mathrm{HO}_{2}+\mathrm{M}$, and $\mathrm{C}_{\mathrm{M}}$ represents the effective thirdbody concentration of reaction $4 f$, which accounts for the non-unity chaperon efficiencies of $\mathrm{H}_{2} \mathrm{O}$ and $\mathrm{H}_{2}$, while

$G=\frac{1+\gamma_{3 b}}{2}+\frac{F}{2}\left\{\left[1+2\left(3+\gamma_{3 b}\right) / F+\left(1+\gamma_{3 b}\right)^{2} / F^{2}\right]^{1 / 2}-1\right\}$,

$H=\frac{1}{2}+\frac{1}{2}\left[1+\frac{4 F k_{7 f}}{\left(k_{5 j}+k_{6 f}\right)} \frac{k_{2 b} k_{2 f}}{\alpha k_{1 b} k_{3 f}}\left(\frac{k_{1 f}}{\alpha k_{4 f} C_{M}}-1\right)\right]^{1 / 2}$,

and

$\alpha=\frac{k_{6 f} F /\left(k_{5 f}+k_{6 j}\right)+G}{F+G}$

are order-unity functions of the composition and temperature [21] involving the reaction-rate constants of the additional reactions $\mathrm{HO}_{2}+\mathrm{H} \stackrel{5 f}{\rightarrow} \mathrm{OH}+\mathrm{OH}, \mathrm{HO}_{2}+\mathrm{H} \stackrel{\text { ff }}{\rightarrow} \mathrm{H}_{2}+\mathrm{O}_{2}$, and $\mathrm{HO}_{2}+\mathrm{OH} \stackrel{\text { f }}{\rightarrow} \mathrm{H}_{2} \mathrm{O}+\mathrm{O}_{2}$, with

$F=\frac{k_{5 f}+k_{6 f}}{k_{7 f}} \frac{k_{3 f}}{k_{4 f} C_{\mathrm{M}}} \frac{\mathrm{Y}_{\mathrm{H}_{2}} / \mathrm{W}_{\mathrm{H}_{2}}}{\mathrm{Y}_{\mathrm{O}_{2}} / \mathrm{W}_{\mathrm{O}_{2}}}$

and

$\gamma_{3 b}=\frac{k_{3 b} Y_{\mathrm{H}_{2} \mathrm{O}} / W_{\mathrm{H}_{2} \mathrm{O}}}{k_{4 f} \mathrm{C}_{\mathrm{M}} \mathrm{Y}_{\mathrm{O}_{2}} / \mathrm{W}_{\mathrm{O}_{2}}}$

The reaction rate given in Eq. (11) applies for temperatures larger than a crossover value $T_{c}$ for which

$k_{1 f}=\alpha k_{4 f} C_{\mathrm{M}}$,

whereas at temperatures $T<T_{c}$ the one-step approximation predicts $\omega=0$ as a consequence of the dissapearance of the radicals. The value of $T_{c}$ depends on the local $\mathrm{H}_{2}$ content through $\alpha$, which equals unity for $Y_{\mathrm{H}_{2}}=0$ because $F=0$ but decreases toward $\alpha=k_{6 f} /\left(k_{5 f}+k_{6 f}\right) \simeq 1 / 6$ with increasing hydrogen concentration. The amount of heat released per unit mass of fuel consumed q, appearing in [6], can be computed from the enthalpy of formation of water vapor $h_{\mathrm{H}_{2} \mathrm{O}}^{\circ}$ according to $q=-h_{\mathrm{H}_{2} \mathrm{O}}^{o} / \mathrm{W}_{\mathrm{H}_{2}}=1.21 \times 10^{5} \mathrm{~kJ} / \mathrm{kg}$.

The radiant heat loss per unit volume is given by the familiar approximation

$\mathrm{Q}_{\mathrm{R}}=4 \kappa_{\mathrm{H}_{2} \mathrm{O}} \sigma p\left(\mathrm{~W} / \mathrm{W}_{\mathrm{H}_{2} \mathrm{O}}\right) \mathrm{Y}_{\mathrm{H}_{2} \mathrm{O}}\left(\mathrm{T}^{4}-\mathrm{T}_{\infty}^{4}\right)$,

where $W$ represents the mean molecular weight of the gas mixture, and $\sigma$ and $\kappa_{\mathrm{H}_{2} \mathrm{O}}$ denote the Stefan-Boltzmann constant and the Plank-mean absorption coefficient, respectively, with the latter being a function of the local temperature [34] that in the range of temperatures of interest $(270<\mathrm{T}<1300 \mathrm{~K})$ can be shown to be accurately represented by the expression $\kappa_{\mathrm{H}_{2} \mathrm{O}}=5.72 \times 10^{-4}(\mathrm{~T} / 298)^{-2} \mathrm{~s}^{2} / \mathrm{kg}$ [20].

As in [20], we shall use the assumption, not strictly necessary, of equal temperature dependence of the diffusivities $D, D_{\mathrm{O}_{2}}$ and $D_{\mathrm{H}_{2} \mathrm{O}}$, so that the two simple relations

$\mathrm{Y}_{\mathrm{H}_{2} \mathrm{O}}-\mathrm{Y}_{\mathrm{H}_{2} \mathrm{O}_{\infty}}=2 \frac{W_{\mathrm{H}_{2} \mathrm{O}}}{W_{\mathrm{O}_{2}}} \frac{D_{\mathrm{O}_{2}}}{D_{\mathrm{H}_{2} \mathrm{O}}}\left(Y_{\mathrm{O}_{2 \infty}}-Y_{\mathrm{O}_{2}}\right)=\frac{W_{\mathrm{H}_{2} \mathrm{O}}}{W_{\mathrm{H}_{2}}} \frac{D}{D_{\mathrm{H}_{2} \mathrm{O}}}\left(\mathrm{Y}_{\mathrm{H}_{2 \infty}}-\mathrm{Y}\right)$

can be readily obtained by integration of linear combinations of Eqs. (3)-(5). In this approximation, the effect of thermal diffusion is incorporated by using in evaluating Eq. (19) an averaged increased diffusivity $D / D_{\mathrm{H}_{2}}=1.154$, obtained from [8] at an average temperature ratio, yielding [20]

$\mathrm{Y}_{\mathrm{H}_{2} \mathrm{O}_{r}}=\mathrm{Y}_{\mathrm{H}_{2} \mathrm{O}_{\omega}}+\frac{W_{\mathrm{H}_{2} \mathrm{O}}}{W_{\mathrm{H}_{2}}} \frac{D}{D_{\mathrm{H}_{2} \mathrm{O}}} \mathrm{Y}_{\mathrm{H}_{2 \infty}}=\mathrm{Y}_{\mathrm{H}_{2} \mathrm{O}_{\omega}}+28.7 \mathrm{Y}_{\mathrm{H}_{2} \omega}$

and

$\frac{Y_{\mathrm{O}_{2 \mathrm{r}}}}{\mathrm{Y}_{\mathrm{O}_{2 \omega}}}=1-\frac{1}{2} \frac{W_{\mathrm{O}_{2}}}{W_{\mathrm{H}_{2}}} \frac{D}{D_{\mathrm{O}_{2}}} \frac{Y_{\mathrm{H}_{2 \omega}}}{Y_{\mathrm{O}_{2 \omega}}}=1-\phi / 0.234$

for the water vapor and oxygen mass fractions in the reaction zone. With these simplifications, the problem reduces to that of integrating

$\frac{1}{r^{2}} \frac{\mathrm{d}}{\mathrm{d} r}\left(\rho \mathrm{Dr} r^{2} \frac{\mathrm{d} Y}{\mathrm{~d} r}\right)=2 \mathrm{~W}_{\mathrm{H}_{2}} \omega$

and

$\frac{1}{r^{2}} \frac{\mathrm{d}}{\mathrm{d} r}\left(\lambda r^{2} \frac{\mathrm{dT}}{\mathrm{d} r}\right)=4 \kappa_{\mathrm{H}_{2} \mathrm{O}} \sigma p_{\mathrm{W}_{\mathrm{H}_{2} \mathrm{O}}}^{\mathrm{W}} \mathrm{Y}_{\mathrm{H}_{2} \mathrm{O}}\left(\mathrm{T}^{4}-\mathrm{T}_{\infty}^{4}\right)-2 \mathrm{~W}_{\mathrm{H}_{2}} q \omega$,

with boundary conditions $\mathrm{dT} / \mathrm{d} r=\mathrm{d} Y / \mathrm{d} r=0$ at $r=0$ and $T-T_{\infty}=Y-Y_{H_{2 \infty}}=0$ as $r \rightarrow \infty$. The expressions given in Eqs. (20) and (21) are to be used in evaluating the reaction rate Eq. (11). As an additional simplification [20], the specific heat at constant pressure can be computed from the approximate formula $c_{p}=c_{p_{\infty}}\left(T / T_{\infty}\right)^{0.15}$, where $c_{p_{\infty}}$ is the value of $c_{p}$ in the ambient atmosphere; this is very accurate for the lean conditions considered and yields $\lambda \propto T^{\gamma}$ and $\rho D \propto T^{\gamma}$ with

$\nu=0.85$ and $\gamma=0.99$

for the temperature dependences of the transport coefficients appearing in Eqs. (22) and (23).

Because of the relatively large activation temperature of reaction $1 f, T_{a_{1 f}}=8590 \mathrm{~K}$, the overall rate $\omega$ given in Eq. (11) is 
very sensitive to changes in temperature through the term $k_{1 f} /\left(\alpha k_{4 f} C_{M}\right)$ appearing in the cutoff factor. The associated value of the dimensionless activation energy

$\beta=T_{a_{1 f}} / T_{c}+n_{1 f}-n_{4 f}+1$,

evaluated at the crossover temperature, with account taken of the additional algebraic temperature dependences $T^{n_{1 f}}$ and $T^{n_{4 f}-1}$ of $k_{1 f}$ and $k_{4 f} C_{M}$, where $n_{1 f}=-0.7$ and $n_{4 f}=-1.4$, is found to be of order 10 . The factor $\rho^{2} k_{2 f} k_{3 f} / k_{1 b}$ in Eq. (11) introduces a nonnegligible additional temperature dependence, characterized by the dimensionless activation energy

$\mu \beta=\left(\mathrm{T}_{a_{2 f}}+\mathrm{T}_{a_{3 f}}-\mathrm{T}_{a_{1 b}}\right) / \mathrm{T}_{\mathrm{c}}+n_{2 f}+n_{3 f}-n_{1 b}-2 \simeq 7$.

Because of these temperature sensivities, the solution exhibits a thin reaction layer centered at $r=r_{f}$ with an apparent peak temperature $T=T_{f}$ that differs by a small relative amount of order $\beta^{-1}$ from the crossover temperature $T_{c}$ along the upper branch of stable solutions [20].

\section{The radiation-free solution}

As a first step in analyzing the solution near the turning point, it is necessary to investigate initially the flame-ball solution that appears in the absence of radiation, the case considered originally by Zeldovich using Arrhenius kinetics with large activation energy [9]. Zeldovich found that, because of the strong temperature dependence of the chemistry, the reaction is confined to a thin layer centered at $r=r_{f}$, where the deficient reactant is consumed, separating a central equilibrium region for $r<r_{f}$ with zero reactant concentration from an outer region for $r>r_{f}$ where the chemical reaction is frozen.

The corresponding radiation-free analysis will be carried out here for the one-step reduced mechanism that describes hydrogen oxidation for temperatures near crossover. Because of the large temperature sensivities of the reaction, the solution exhibits also in this case the characteristic thin-reactionlayer structure found in the Zeldovich analysis. A remarkable difference with the previous work is that, while the Arrhenius kinetics employed by Zeldovich provides no flammability limit, the presence of the factor $k_{1 f} /\left(\alpha k_{4 f} C_{M}\right)-1$ in the nonArrhenius rate (11) introduces a kinetically controlled flammability limit for hydrogen-air combustion, associated with the limiting mixture conditions for which the peak temperature equals the crossover temperature $T_{c}$.

The analysis with radiation neglected begins by integrating twice a chemistry-free linear combination of Eqs. (22) and (23) to yield

$\frac{L_{\mathrm{H}_{2}} c_{p \infty} T_{\infty}}{1+\nu-\gamma}\left(\frac{T}{T_{\infty}}\right)^{1+y-\gamma}+q \mathrm{Y}=\frac{L_{\mathrm{H}_{2}} c_{p \infty} T_{\infty}}{1+\nu-\gamma}+q \mathrm{Y}_{\mathrm{H}_{2 \infty}}$

which can be evaluated at the flame, where $Y=0$ to give an expression determining the flame-ball temperature

$\left(\frac{T_{f}}{T_{\infty}}\right)^{1+\nu-\gamma}=1+\frac{(1+\nu-\gamma) q Y_{\mathrm{H}_{2 \infty}}}{\mathrm{L}_{\mathrm{H}_{2}} \mathrm{C}_{p \infty} \mathrm{T}_{\infty}}$

For $r>r_{f}$ the reaction is frozen and one may integrate Eq. (22) with boundary conditions $Y=0$ at $r=r_{f}$ and $Y=Y_{\mathrm{H}_{2 \infty}}$ at $r=\infty$ to give the diffusive flux of hydrogen into the reaction sheet, from

$\left(\frac{\mathrm{d} Y}{\mathrm{~d} r}\right)_{r_{f+}}=\frac{1+\nu-\gamma}{1+\nu} \frac{\left(\mathrm{T}_{f} / \mathrm{T}_{\infty}\right)^{1+\nu}-1}{\left(\mathrm{~T}_{f} / \mathrm{T}_{\infty}\right)^{\gamma}\left[\left(\mathrm{T}_{f} / \mathrm{T}_{\infty}\right)^{1+\nu-\gamma}-1\right]} \frac{\mathrm{Y}_{\mathrm{H}_{2 \infty}}}{r_{f}}$,

which is consumed according to

$\frac{(\rho D)_{f}}{4 W_{H_{2}}}\left(\frac{d Y}{d r}\right)_{r_{f+}}^{2}=\int_{0}^{\infty} \omega d Y$

obtained from integration of the quasi-planar form of Eq. (22) across the thin reaction layer after multiplication by $d Y / d r$, with the value of $(\rho D)_{f}$ evaluated at the flame temperature. The reaction rate $\omega$ appearing in the above integral is given in Eq. (11). In performing the integral, use must be made of Eq. (27) to give $T$ as a function of $Y$, while the constant reactionsheet values $\mathrm{Y}_{\mathrm{H}_{2} \mathrm{O}_{\mathrm{r}}}$ and $\mathrm{Y}_{\mathrm{O}_{2 \mathrm{r}}}$ given in Eqs. (20) and (21) can be used to evaluate the water-vapor and oxygen mass fractions. Equations (29) and (30) determine the flame-ball radius $r_{f}$ once the peak temperature is obtained from Eq. (28). We shall see below that this radiation-free analysis provides the leadingorder solution for hydrogen-air flame balls in the double asymptotic limit of large activation energy and small radiation losses, to be presented below in dimensionless form, so that, for instance, Eq. (30) is to be rewritten in Eq. (43) in nondimensional form.

The analysis described above fails as the peak temperature decreases to the crossover value $T_{c}$, defined from Eq. (17), corresponding to a critical value of the fuel mass fraction

$\mathrm{Y}_{\mathrm{H}_{2 \infty \mathrm{I}}}^{o}=\frac{\mathrm{L}_{\mathrm{H}_{2}}\left[\left(\mathrm{~T}_{\mathrm{c}} / \mathrm{T}_{\infty}\right)^{1+\nu-\gamma}-1\right]}{(1+\nu-\gamma) q /\left(\mathrm{c}_{p \infty} \mathrm{T}_{\infty}\right)}$.

As $\mathrm{Y}_{\mathrm{H}_{2} \infty} \rightarrow \mathrm{Y}_{\mathrm{H}_{2 \omega 1}}^{o}$ the reaction rate $\omega$ diminishes, resulting in a vanishing value of $(\mathrm{dY} / \mathrm{d} r)_{f+}$ for $\mathrm{Y}_{\mathrm{H}_{2 \omega}}=\mathrm{Y}_{\mathrm{H}_{2 \omega \mathrm{I}}}^{\mathrm{D}}$ as dictated by Eq. (30) and a corresponding diverging value of $r_{f} \rightarrow \infty$ as given by Eq. (29). Clearly, the expression given in Eq. (31) provides a first-order estimate of the critical $\mathrm{H}_{2}$ mass fraction at the flammability limit. In the evaluation, the crossover temperature $T_{c}=T_{c}^{o}$ must be computed from

$k_{1 f}=k_{4 j} C_{M}$,

obtained from Eq. (17) with $\alpha=1$, as corresponds to the vanishing $\mathrm{H}_{2}$ concentration found in the reaction layer at the flammability limit. Since the effective third-body efficiency $[27,28]$

$\mathrm{C}_{\mathrm{M}}=\frac{p}{\mathrm{R}^{\circ} \mathrm{T}}\left(1+15 \mathrm{Y}_{\mathrm{H}_{2} \mathrm{O}_{\mathrm{r}}} \mathrm{W} / \mathrm{W}_{\mathrm{H}_{2} \mathrm{O}}\right)$

depends on the water-vapor mass fraction, given in Eq. (20) as a function of $Y_{\mathrm{H}_{2 \infty}}$, Eqs. (31) and (32) are coupled and need to be solved simultaneously to determine $\mathrm{T}_{\mathrm{c}}^{o}$ and $\mathrm{Y}_{\mathrm{H}_{2 \infty 1}}^{o}$.

Note that, because of the small value of the Lewis number $\mathrm{L}_{\mathrm{H}_{2}}=0.3$, the peak temperature at the reaction layer given in Eq. (28) is a factor $\mathrm{L}_{\mathrm{H}_{2}}^{-1}$ larger than the adiabatic flame temperature corresponding to the planar flame. Correspondingly, the critical $\mathrm{H}_{2}$ content at extinction, given in Eq. (31), is a factor $\mathrm{L}_{\mathrm{H}_{2}}$ smaller than its planar-flame counterpart, a consequence of preferential diffusion first pointed out by Joulin [35]. For 
instance, for a $\mathrm{H}_{2}$-air mixture at $\mathrm{T}_{\infty}=300 \mathrm{~K}$ and $p=1 \mathrm{~atm}$, solving Eqs. (31) and (32) yields $\mathrm{T}_{\mathrm{c}}^{\circ}=1049 \mathrm{~K}$ and $\mathrm{Y}_{\mathrm{H}_{2 \infty 1}}^{\circ}=0.00173$, giving at this order $\phi_{1}^{0}=0.059$ for the equivalence ratio at the lean flammability limit, to be compared with the value $\phi_{1}=0.251$ obtained from planar-deflagration computations $[21,22]$. Also of interest is that the temperature dependence of the transport coefficients (including Soret effects) is present in the solution at this order through the non-unity factor $1+\nu-\gamma$ appearing in Eq. (31), needed for increased accuracy. The appearance of the turning point, as a result of the nearcrossover competition between effects of radiation and finite-rate kinetics, will next be seen to introduce a correction to the leading-order prediction given in Eq. (31)

\section{Characteristic scales near the turning point}

The characteristic flame-ball size near the turning point can be estimated by anticipating that the transition between the two branches of solutions occurs when the peak temperature differs from the crossover value by a small amount of order $\mathrm{T}_{f}-\mathrm{T}_{c} \sim \beta^{-1} \mathrm{~T}_{C}$, with $\mathrm{Y} \sim \beta^{-1} \mathrm{Y}_{\mathrm{H}_{2 \infty}}$ in the reaction layer. Since the diffusive flux of hydrogen is of order $\mathrm{dY} / \mathrm{d} r \sim \mathrm{Y}_{\mathrm{H}_{2 \infty}} / r_{0}$, with $r_{0}$ representing the characteristic flame-ball radius, the reactionlayer thickness can be expected to be of order $\beta^{-1} r_{0}$. Using these estimates in Eq. (30) with use made of Eq. (11) to evaluate $\omega$ yields

$r_{0}=\left(\frac{\beta^{3} D_{\mathrm{c}} G_{\mathrm{c}} H_{\mathrm{c}}}{2\left(k_{2 f} k_{3 f} / k_{1 b}\right)_{\mathrm{c}}\left(\rho_{\mathrm{c}} Y_{\mathrm{H}_{2 \sigma}} / W_{\mathrm{H}_{2}}\right)}\right)^{1 / 2}$

for the characteristic flame-ball radius near the turning point, where the subscript $c$ denotes quantities evaluated at the crossover temperature $\mathrm{T}_{c}^{o}$, also used in evaluating $\beta$ from Eq. (25).

Flame balls near the turning point are sufficiently small for radiation to be relatively weak, as can be seen by evaluating the order-of-magnitude ratio $O\left(Q_{R}\right) / O(\nabla \cdot(\lambda \nabla T)$ ), involving the characteristic magnitudes of the volumetric heat losses by radiation and conduction. When the ambient atmosphere is free from water vapor, it is appropriate to use the characteristic reaction-layer value $\mathrm{Y}_{\mathrm{H}_{2} \mathrm{O}_{r}}=28.7 \mathrm{Y}_{\mathrm{H}_{20}}$ computed from Eq. (20) to evaluate Eq. (18), thereby yielding the radiation-toconduction ratio

$\varepsilon=4 \kappa_{\mathrm{H}_{2} \mathrm{O}_{\mathrm{c}}} \sigma p\left(\mathrm{~W}_{\infty} / \mathrm{W}_{\mathrm{H}_{2} \mathrm{O}}\right) \mathrm{Y}_{\mathrm{H}_{2} \mathrm{O}_{\mathrm{r}}} \mathrm{T}_{c}^{3} r_{0}^{2} / \lambda_{\mathrm{c}}$.

If water vapor is present in the atmosphere, however, then the alternative parameter

$\varepsilon_{\mathrm{W}}=4 \kappa_{\mathrm{H}_{2} \mathrm{O}_{\mathrm{c}}} \sigma p\left(\mathrm{~W}_{\infty} / \mathrm{W}_{\mathrm{H}_{2} \mathrm{O}}\right) \mathrm{Y}_{\mathrm{H}_{2} \mathrm{O}_{\infty}} \mathrm{T}_{\mathrm{c}}^{3} r_{0}^{2} / \lambda_{\mathrm{c}}$

is a more appropriate measure of the radiation effect. Both $\varepsilon$ and $\varepsilon_{W}$ typically take on small values. For instance, the parameter $\varepsilon$, a function of the temperature, gives $\varepsilon \simeq 0.05$, independent of $Y_{\mathrm{H}_{2 \infty}}$, when evaluated at $T_{\mathrm{c}} \simeq 1000 \mathrm{~K}$ with the ambient mean molecular weight $W_{\infty}$ taken to be equal to that of air. As a result, radiation is unimportant, at leading order, at distances of order $r_{0}$, and it only becomes significant far from the flame ball, at large distances, of order $\varepsilon^{-1} r_{0}$ for $\mathrm{Y}_{\mathrm{H}_{2} \mathrm{O}_{\omega}}=0$ and $\varepsilon_{W}^{-1 / 2} r_{0}$ for $\mathrm{Y}_{\mathrm{H}_{2} \mathrm{O}_{\infty}} \neq 0$. Its effect in the far field is that of introducing an apparent ambient temperature $T_{\infty}^{*}$ for the description of the temperature field at distances of order $r_{0}$. For the values of $\varepsilon$ and $\varepsilon_{\mathrm{W}}$ typically encountered, the characteristic values of the corresponding relative temperature decrements, given by $\left(T_{\infty}-T_{\infty}^{*}\right) / T_{c} \sim \varepsilon \ln \left(\varepsilon^{-1}\right)$ when $\mathrm{Y}_{\mathrm{H}_{2} \mathrm{O}_{\infty}}=0$ and by $\left(\mathrm{T}_{\infty}-\mathrm{T}_{\infty}^{*}\right) / \mathrm{T}_{\mathrm{c}} \sim \varepsilon_{\mathrm{W}}^{1 / 2}$ when $\mathrm{Y}_{\mathrm{H}_{2} \mathrm{O}_{\mathrm{co}}} \neq 0$, turn out to be of order $\beta^{-1}$, and are therefore large enough to affect the solution at leading order, modifying the flame-ball radius and enabling the transition to the upper branch through a turning point in the $r_{f}-\phi$ curve to occur. These observations suggest the use of the distinguished limit $\beta \varepsilon \ln \left(\varepsilon^{-1}\right) \sim O(1)$ (or $\beta \varepsilon_{W}^{1 / 2} \sim O(1)$ ) for the asymptotic treatment of the flame-ball problem for $\beta \gg 1$ and $\varepsilon \ll 1$ (or $\left.\varepsilon_{W} \ll 1\right)$.

\section{Solution for $\beta \gg 1$ with $\varepsilon \ll 1\left(\varepsilon_{\mathrm{W}} \ll 1\right)$ \\ 6.1. Governing equations}

Introducing the dimensionless variables $\xi=r / r_{0}, \theta=\mathrm{T} / \mathrm{T}_{C}$, and $y=Y / Y_{\mathrm{H}_{2 \infty}}$, reduces Eqs. (22) and (23) to

$\frac{1}{\xi^{2}} \frac{\mathrm{d}}{\mathrm{d} \xi}\left(\theta^{\gamma} \xi^{2} \frac{\mathrm{dy}}{\mathrm{d} \xi}\right)=\Omega$

and

$\frac{1}{\xi^{2}} \frac{\mathrm{d}}{\mathrm{d} \xi}\left(\theta^{y} \xi^{2} \frac{\mathrm{d} \theta}{\mathrm{d} \xi}\right)=\tilde{\mathrm{Q}}_{R}-\tilde{q} \Omega$

where

$\tilde{q}=\frac{q Y_{\mathrm{H}_{2 \infty}}}{L_{\mathrm{H}_{2}}\left(\mathrm{~T}_{\mathrm{c}}^{0} / \mathrm{T}_{\infty}\right)^{1+\nu-\gamma} c_{p \infty} T_{\infty}}$,

$\tilde{Q}_{R}=\left\{\begin{array}{lr}\varepsilon(1-y)\left(\theta^{4}-\theta_{\infty}^{4}\right) / \theta^{2} & \text { for } \mathrm{Y}_{\mathrm{H}_{2} \mathrm{O}_{\infty}}=0 \\ \varepsilon_{\mathrm{W}}\left[1+28.7\left(\mathrm{Y}_{\mathrm{H}_{2 \infty}} / \mathrm{Y}_{\mathrm{H}_{2} \mathrm{O}_{\infty}}\right)(1-y)\right]\left(\theta^{4}-\theta_{\infty}^{4}\right) / \theta^{2} & \text { for } \mathrm{Y}_{\mathrm{H}_{2} \mathrm{O}_{\infty}}>0\end{array}\right.$

and

$\Omega=\beta^{3} \exp \left(\mu \beta \frac{\theta-1}{\theta}\right)\left[\exp \left(\beta \frac{\theta-1}{\theta}\right)-1\right] \mathrm{y}^{2}$,

for $\theta>1$ while $\Omega=0$ for $\theta \leq 1$. Appropriate boundary conditions are $\mathrm{dy} / \mathrm{d} \xi=\mathrm{d} \theta / \mathrm{d} \xi=0$ at $\xi=0$ and $\mathrm{y}-1=\theta-\theta_{\infty}=0$ as $\xi \rightarrow \infty$. It is clear from the above dimensionless problem that in the limit $\varepsilon \ll 1$ (i.e., $\varepsilon_{W} \ll 1$ ) radiation is negligible at distances of order $\xi \sim O(1)$ and that the chemical term balances the diffusion and conduction terms in the reaction layer, of thickness $\beta^{-1}$, where $\mathrm{y} \sim \beta^{-1}$ and $\theta-1 \sim \beta^{-1}$.

\subsection{Near-field solution}

In the limit $\beta \rightarrow \infty$ the reaction is confined to a thin sheet located at $\xi=\xi_{f}$, across which the gradients of temperature and fuel mass fraction are related by the jump condition

$\left(\frac{\mathrm{d} \theta}{\mathrm{d} \xi}\right)_{\xi_{f}}-\left(\frac{\mathrm{d} \theta}{\mathrm{d} \xi}\right)_{\xi_{f_{+}}}=\tilde{q} \theta_{f}^{\gamma-\nu}\left(\frac{\mathrm{dy}}{\mathrm{d} \xi}\right)_{\xi_{f_{+}}}$.

A second equation determining the burning rate at the flame comes from integrating Eq. (37) across the reaction layer to give 
$\frac{1}{2} \theta_{f}^{\gamma}\left(\frac{\mathrm{d} y}{\mathrm{~d} \xi}\right)_{\xi_{f_{+}}}^{2}=\int_{0}^{y_{c}} \Omega \mathrm{dy}$

the dimensionless counterpart of Eq. (30). Computation of the integral requires use of $\theta_{f}-\theta=\tilde{q} \theta_{f}^{\gamma-\nu} y$ to evaluate the reaction rate $\Omega$ given in Eq. (41), with the condition $\Omega=0$ for $\theta \leq 1$ introducing an upper limit of integration

$\mathrm{y}_{\mathrm{c}}=\left(\theta_{f}-1\right) /\left(\tilde{q} \theta_{f}^{\gamma-\nu}\right)$,

associated with the fuel concentration at the location where $\theta=1$, a quantity of order $\beta^{-1}$.

Since $\varepsilon \ll 1$, the solution in the near field, corresponding to distances $\xi \sim O(1)$, can be determined at leading order with radiation neglected to obtain results with errors of order $\varepsilon$. Integration of Eqs. (37) and (38) with the preceding conditions then yields simply $\theta-\theta_{f}=y=0$ for $\xi<\xi_{f}$, and

$\theta^{1+\nu^{\prime}}=\theta_{f}^{1+\nu}-\left[\theta_{f}^{1+\nu^{\prime}}-\left(\theta_{\infty}^{*}\right)^{1+\nu^{\prime}}\right]\left(1-\frac{\xi_{f}}{\xi}\right)$

and

$1-\mathrm{y}=\frac{\theta^{1+\gamma^{*}-\gamma}-\left(\theta_{\infty}^{*}\right)^{1+\nu-\gamma}}{\theta_{f}^{1+\gamma^{-\gamma}-}-\left(\theta_{\infty}^{*}\right)^{1+\nu-\gamma}}$

for $\xi>\xi_{f}$. In anticipation of the fact that the effect of radiation modifies the far-field temperature profile in Eqs. (45) and (46), an apparent ambient temperature $\theta_{\infty}^{*}$ has been introduced to replace $\theta_{\infty}$ as the boundary condition for $\xi \rightarrow \infty$. Eq. (46) gives the gradient

$\left(\frac{\mathrm{dy}}{\mathrm{d} \xi}\right)_{\xi_{f+}}=-\frac{(1+\nu-\gamma) \theta_{f}^{\gamma-\gamma}}{\theta_{f}^{1+\nu-\gamma}-\left(\theta_{\infty}^{*}\right)^{1+\nu-\gamma}}\left(\frac{\mathrm{d} \theta}{\mathrm{d} \xi}\right)_{\xi_{f+}}$,

where

$\left(\frac{\mathrm{d} \theta}{\mathrm{d} \xi}\right)_{\xi_{f+}}=-\frac{\theta_{f}^{1+\nu}-\left(\theta_{\infty}^{*}\right)^{1+\nu}}{(1+\nu) \theta_{f}^{y} \xi_{f}}$

is determined from Eq. (45). Since $(\mathrm{d} \theta / \mathrm{d} \xi)_{f-}=0$ (i.e., $\theta=\theta_{f}$ for $\xi<\xi_{f}$ ), substitution of Eq. (47) into Eq. (42) provides the relationship

$\tilde{q}=\frac{\theta_{f}^{1+\nu-\gamma}-\left(\theta_{\infty}^{*}\right)^{1+\nu-\gamma}}{1+\nu-\gamma}$.

On the other hand, using Eqs. (47) and (48) to express $(\mathrm{dy} / \mathrm{d} \xi)_{\xi_{f+}}$ reduces Eq. (43) to

$\frac{1}{2 \theta_{f}^{\gamma}}\left(\frac{1+\nu-\gamma}{1+\nu}\right)^{2}\left(\frac{\theta_{f}^{1+\nu}-\left(\theta_{\infty}^{*}\right)^{1+\nu}}{\theta_{f}^{1+\nu-\gamma}-\left(\theta_{\infty}^{*}\right)^{1+\nu-\gamma}}\right)^{2} \frac{1}{\xi_{f}^{2}}=\int_{0}^{y_{c}} \Omega d y$.

The turning point appears for values of $Y_{\mathrm{H}_{2 \infty}}$ close to $\mathrm{Y}_{\mathrm{H}_{2 \infty}}^{o}$ and corresponding values of $\theta_{f}$ close to unity. It is then appropriate to linearize Eq. (49) for $\bar{q}-\left(1-\theta_{\infty}^{1+\gamma-\gamma}\right)$ / $(1+\nu-\gamma) \ll 1$ to yield the approximate expression

$\tilde{q}-\frac{1-\theta_{\infty}^{1+\nu-\gamma}}{1+\nu-\gamma}=\left(\theta_{f}-1\right)+\left(\theta_{\infty}-\theta_{\infty}^{*}\right)$.

For the description of the turning point in the limit $\beta \gg 1$, it is convenient to introduce the reduced variables
$\Theta_{f}=\beta\left(\theta_{f}-1\right)$

and

$\mathrm{Q}=\beta\left(\tilde{q}-\frac{1-\theta_{\infty}^{1+\nu-\gamma}}{1+\nu-\gamma}\right)$.

Note that $\left(1-\theta_{\infty}^{1+\nu-\gamma}\right) /(1+\nu-\gamma)$ is the value of $\tilde{q}$ evaluated with the limiting fuel mass fraction $\mathrm{Y}_{\mathrm{H}_{2 \omega 1}}^{0}$, as can seen from Eqs. (31) and (39), so that

$\mathrm{Q}=\beta \frac{1-\theta_{\infty}^{1+y-\gamma}}{1+\nu-\gamma} \frac{\left(\mathrm{Y}_{\mathrm{H}_{2 \omega}}-\mathrm{Y}_{\mathrm{H}_{2 \omega l}}^{o}\right)}{\mathrm{Y}_{\mathrm{H}_{2 \propto 1}}^{0}}$,

can be seen as a reduced equivalence ratio measuring the departures of the mixture fuel content from the critical value corresponding to the radiation-free flammability limit. In terms of $\Theta_{f}$ and $Q$ the expression given in Eq. (51) reduces to

$\mathrm{Q}=\Theta_{f}+\beta\left(\theta_{\infty}-\theta_{\infty}^{*}\right)$,

establishing a balance between the non-dimensional heat release by chemical reaction $Q$ the non-dimensional temperature increment $\Theta_{f}$, and the non-dimensional farfield heat losses by radiation, $\beta\left(\theta_{\infty}-\theta_{\infty}^{*}\right)$. At the same level of approximation, the reaction-rate integral in Eq. (50) can be evaluated explicitly, and the result is that Eq. (50) can be written for near-turning-point conditions in terms of the normalized radius

$\mathrm{R}_{f}=\frac{(1+\nu)(1+\nu-\gamma)^{1 / 2}}{\left(1-\theta_{\infty}^{1+\gamma^{\prime}}\right)\left(1-\theta_{\infty}^{1+\gamma-\gamma}\right)^{1 / 2}} \xi_{j}$,

as

$\frac{1}{R_{f}^{2}}=4 e^{\mu \Theta_{f}}\left(\frac{e^{\Theta_{f}}}{(\mu+1)^{3}}-\frac{1}{\mu^{3}}\right)+\frac{2 \Theta_{f}^{2}}{\mu(\mu+1)}+\frac{4 \Theta_{f}(1+2 \mu)}{\mu^{2}(\mu+1)^{2}}+\frac{4\left(3 \mu^{2}+3 \mu+1\right)}{\mu^{3}(\mu+1)^{3}}$.

In this expansion the value $\mu=0.7$ will be used below for computational purposes (see the discussion following Eq. (25)). Note that in the limit of large activation energy considered here, the dimensionless variables $\Theta_{f}, Q$ and $R_{f}$ of order unity, defined in Eqs. (52), (54), and (56), are appropriately scaled measures for $\mathrm{T}_{f}, \phi$ and $r_{f}$ near the turning point. Correspondingly, the curves to be obtained below for the variation of $R_{f}$ and $\Theta_{f}$ with $Q$ shown in Fig. 3, are merely an appropriately rescaled representation near the turning point of the corresponding dimensional plots shown in Figs. 1 and 2.

Equations (55) and (57) determine $R_{f}$ and $\Theta_{f}$ for a given value of $\beta\left(\theta_{\infty}-\theta_{\infty}^{*}\right)$. This apparent ambient-temperature modification is determined from matching the temperature profile given in Eq. (45), which as $\xi \rightarrow \infty$ takes the form

$\theta-\theta_{\infty}=\frac{1-\theta_{\infty}^{1+\nu}}{(1+\nu) \theta_{\infty}^{\nu}} \frac{\xi_{f}}{\xi}-\left(\theta_{\infty}-\theta_{\infty}^{*}\right)$

with the solution in the far-field region, where radiation enters at leading order in determining the final decay toward the boundary value $\theta_{\infty}$. The far-field solution is different depending on whether or not the ambient atmosphere contains water vapor, leading to different expressions for the turning-point curves. 


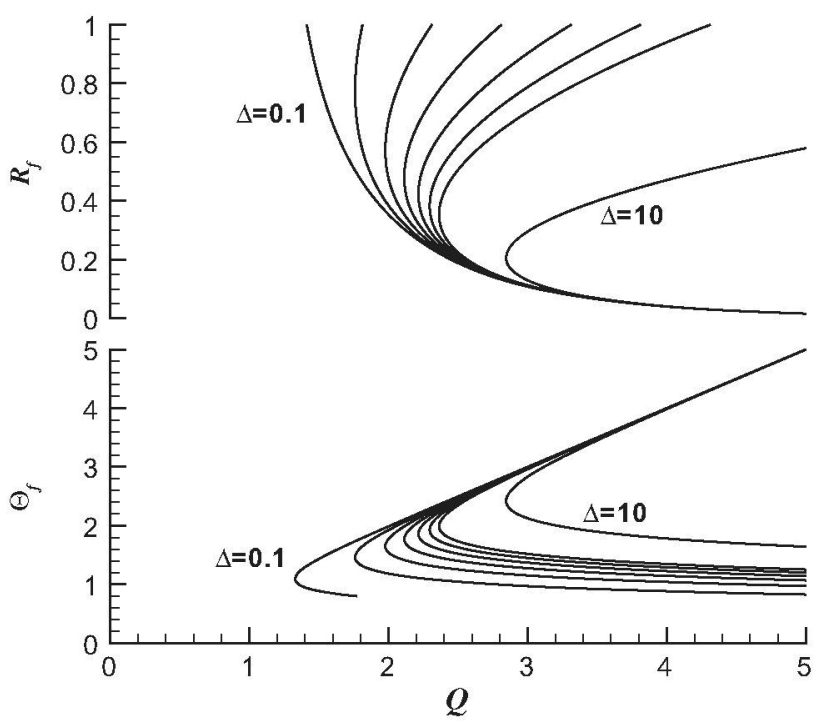

Fig. 3 - The variation of the scaled non-dimensional flameball radius $R_{f}$ and of the reduced non-dimensional flameball temperature $\Theta_{f}$ with the appropriate reduced equivalence ratio $Q$ as obtained from Eqs. (57) and (64) for values of the relevant non-dimensional radiant heat-loss parameter (65) $\Delta=(0.1,0.5,1,1.5,2,2.5,3,10)$.

\subsection{The turning point for $\mathrm{Y}_{\mathrm{H}_{2} \mathrm{O}_{\infty}}=0$}

With the water-vapor concentration decaying in the far field according to $1-y \propto 1 / \xi$, radiation is seen to become comparable to conduction at large distances of order $\xi \sim \varepsilon^{-1}$, where $\theta-\theta_{\infty} \sim \varepsilon$. The solution is obtained by integrating

$\frac{\mathrm{d}}{\mathrm{d} \eta}\left(\eta^{2} \frac{\mathrm{d} \tilde{\theta}}{\mathrm{d} \eta}\right)=\Lambda \eta \tilde{\theta} / \xi_{f}, \begin{cases}\eta \rightarrow 0: & \eta^{2} \mathrm{~d} \tilde{\theta} / \mathrm{d} \eta \rightarrow-\xi_{f} \\ \eta \rightarrow \infty: & \tilde{\theta} \rightarrow 0\end{cases}$

where $\eta=\varepsilon \xi$,

$\tilde{\theta}=\frac{(1+\nu) \theta_{\infty}^{\nu}}{1-\theta_{\infty}^{1+\nu}} \frac{\theta-\theta_{\infty}}{\varepsilon}$

and

$\Lambda=4 \frac{1+\nu-\gamma}{1+\nu} \frac{\theta_{\infty}^{1-\nu-\gamma}\left(1-\theta_{\infty}^{1+\nu}\right)}{\left(1-\theta_{\infty}^{1+\nu-\gamma}\right)} \xi_{f}^{2}$.

As $\eta \rightarrow 0$ the solution of Eq. (59) is seen to be

$\tilde{\theta}=\xi_{f} / \eta+\Lambda \ln \eta$,

so that matching with the inner solution given in Eq. (58) provides in this case

$\theta_{\infty}-\theta_{\infty}^{*}=\frac{\Lambda\left(1-\theta_{\infty}^{1+\nu}\right)}{(1+\nu) \theta_{\infty}^{\nu}} \varepsilon \ln \left(\varepsilon^{-1}\right)$

to be substituted into Eq. (55) to yield

$\mathrm{Q}=\Theta_{f}+\Delta R_{f}^{2}$, where

$\Delta=\frac{4 \theta_{\infty}^{1-2 v-\gamma}\left(1-\theta_{\infty}^{1+\gamma^{\prime}}\right)^{4}}{(1+\nu)^{4}} \beta \varepsilon \ln \left(\varepsilon^{-1}\right)$.

The solution then depends on the radiation parameter, $\Delta$, of order unity. Sample plots showing the variations of the normalized flame-ball radius $R_{f}$ and the reduced peak temperature $\Theta_{f}$, as functions of the reduced equivalence ratio Q obtained from simultaneously solving Eqs. (57) and (64), are shown in Fig. 3 for different values of $\Delta$. As can be seen, two solutions are found for $Q>Q$, and no solution exist for $\mathrm{Q}<\mathrm{Q}$. For $\mathrm{Q} \rightarrow \infty$, along the upper branch the radius increases and the peak temperature approaches crossover according to $R_{f} \simeq(Q / \Delta)^{1 / 2}$ and, since the first four terms vanish in the expansion of Eq. (57) for small $\Theta_{f}, \Theta_{f} \simeq(6 \Delta / Q)^{1 / 4}$. The solution therefore evolves to approach that previously given in [20] for flame balls away from the turning point, with the flame-ball radius determined by the condition that radiant heat losses remove enough of the heat released by chemical reaction at the flame to keep $T_{f}=T_{c}$. On the other hand, the solution for the Zeldovich lower branch, with negligible radiation, increasing temperature, and vanishing radius, reduces to $\Theta_{f} \simeq Q$ and $R_{f} \simeq \frac{1}{2}(1+\mu)^{3 / 2} \exp [-(1+\mu) Q / 2]$. The numerical computations shown in Figs. 1 and 2 indicate that this last asymptotic solution fails away from the turning point, with the temperature eventually decreasing after reaching a maximum. This failure occurs when $r_{f}$ reaches small values, indicating that for small flame balls in richer mixtures the chemical reaction no longer occurs in a thin layer where the fuel is completely consumed, but rather in a central region with incomplete hydrogen consumption.

\subsection{The turning point for $\mathrm{Y}_{\mathrm{H}_{2} \mathrm{O}_{\infty}}>0$}

When water vapor is present in the surrounding atmosphere radiation losses enter to modify the temperature field at relative distances of order $\xi \sim \varepsilon_{\mathrm{W}}^{-1 / 2}$, where $\theta-\theta_{\infty} \sim \varepsilon_{\mathrm{W}}^{1 / 2}$, so that $\varepsilon_{\mathrm{W}}^{1 / 2}$ must replace $\varepsilon$ in the definitions of $\eta$ and $\tilde{\theta}$. The solution for the temperature profile, $\tilde{\theta}=\xi_{f} \exp \left[-2 \theta_{\infty}^{(1-v) / 2} \eta\right] / \eta$, obtained by integrating

$\frac{1}{\eta^{2}} \frac{\mathrm{d}}{\mathrm{d} \eta}\left(\eta^{2} \frac{\mathrm{d} \tilde{\theta}}{\mathrm{d} \eta}\right)=4 \theta_{\infty}^{1-\nu} \tilde{\theta},\left\{\begin{array}{ll}\eta \rightarrow 0: & \eta^{2} \mathrm{~d} \tilde{\theta} / \mathrm{d} \eta \rightarrow-\xi_{f}, \\ \eta \rightarrow \infty: & \tilde{\theta} \rightarrow 0\end{array}\right.$,

takes the limiting form

$\tilde{\theta}=\xi_{f} / \eta-2 \theta_{\infty}^{(1-\nu) / 2} \xi_{f}$

as $\eta \rightarrow 0$, so that matching with the inner solution given in Eq. (58), used already in Eq. (66) when writing the boundary condition as $\eta \rightarrow 0$, provides

$\theta_{\infty}-\theta_{\infty}^{*}=\frac{2\left(1-\theta_{\infty}^{1+\nu}\right) \theta_{\infty}^{(1-3 \nu) / 2}}{(1+\nu)} \varepsilon_{\mathrm{W}}^{1 / 2} \xi_{f}$.

Substitution of this last expression into Eq. (55) yields

$\mathrm{Q}=\Theta_{f}+\Delta_{W} R_{f}$,

with the revised radiation parameter of order unity being 
$\Delta_{\mathrm{W}}=\frac{2 \theta_{\infty}^{(1-3 r) / 2}\left(1-\theta_{\infty}^{1+\nu}\right)^{2}\left(1-\theta_{\infty}^{1+\nu-\gamma}\right)^{1 / 2}}{(1+\nu)^{2}(1+\nu-\gamma)^{1 / 2}} \beta \varepsilon_{\mathrm{W}}^{1 / 2}$.

When the ambient atmosphere contains water vapor, the variation of the normalized flame-ball radius $R_{f}$ and the reduced peak temperature $\Theta_{f}$, as functions of $Q$ are obtained by solving simultaneously Eqs. (57) and (69) for different values of $\Delta_{W}$. The solution is qualitatively similar to that depicted in Fig. 3 for dry ambient conditions, the only difference arising from the linear functional dependence on $R_{f}$ in Eq. (69), rather than the quadratic dependence in Eq. (64). As before, two branches of solutions exist for $Q>Q_{J}$, with the solution along the branch of decreasing radii away from the turning point approaching the radiation-free solution $\Theta_{f}=Q$ and $R_{f}=\frac{1}{2}(1+\mu)^{3 / 2} \exp [-(1+\mu) Q / 2]$, whereas the branch of increasing radii approaches for $Q \gg 1$ the asymptotic form $R_{f}=Q / \Delta_{W}$ and $\Theta_{f}=6^{1 / 4}\left(\Delta_{W} / Q\right)^{1 / 2}$.

\section{Limiting conditions for flame-ball existence}

For given conditions of pressure, ambient temperature and composition, Eqs. (31) and (32) can be used to obtain the values of $\mathrm{T}_{\mathrm{c}}^{\circ}$ and $\mathrm{Y}_{\mathrm{H}_{2 \infty \mathrm{l}}}^{\mathrm{O}}$, with the water-vapor mass fraction given in Eq. (20) used to evaluate the third-body efficiency present in Eq. (33). This leading-order prediction for the critical conditions can be improved by using the results of the turning-point analysis, which, according to the definition given in Eq. (54), yields

$Y_{\mathrm{H}_{2 \infty 1}}=\mathrm{Y}_{\mathrm{H}_{2 \infty 1}}^{o}\left(1+\frac{(1+\nu-\gamma) \mathrm{Q}_{\mathrm{J}}}{\beta\left(1-\theta_{\infty}^{1+\gamma^{-\gamma}}\right)}\right)$

as the first-order correction. Equation (25) and $\theta_{\infty}=T_{\infty} / T_{c}$ evaluated with $\mathrm{T}=\mathrm{T}_{c}=\mathrm{T}_{\mathrm{c}}^{o}$ provide the values of $\beta$ and $\theta_{\infty}$, whereas Eq. (35) (or Eq. (36) if water is present in the atmosphere) gives the value of $\varepsilon$ (or $\varepsilon_{W}$ ), to be used in evaluating $\Delta$ from Eq. (65) (or $\Delta_{W}$ from Eq. (70)). For instance, for the dry conditions considered in Figs. 1 and 2 the value $T_{c}^{o}=1049 \mathrm{~K}$ previously computed gives $\beta=9.9$ and $\theta_{\infty}=0.286$, so that $\varepsilon=0.044$ and $\Delta=2.54$. This last value could be used when selecting the curves $R_{f}-Q$ and $\Theta_{f}-Q$ in Fig. 3 for the nearturning-point variation of the flame-ball radius and temperature (the non-dimensional representation of the curves shown in Figs. 1 and 2), which in turn determine the critical value $Q_{I}=$ 2.3 appearing in Eq. (71), thereby giving $\mathrm{Y}_{\mathrm{H}_{2 \omega 1}}=0.00225$ $\left(\phi_{1}=0.0774\right)$ as a correction to the radiation-free prediction $\mathrm{Y}_{\mathrm{H}_{2 \infty}}^{o}=0.00173\left(\phi_{1}^{0}=0.059\right)$. In general, the simple expressions

$\mathrm{Q}_{\mathrm{I}}=-0.525+2.50 \Delta^{0.13}$

and

$\mathrm{Q}_{\mathrm{l}}=-0.609+2.83 \Delta_{\mathrm{W}}^{0.22}$,

obtained by fitting the numerical solution of Eqs. (57) and (64) (or Eq. (69) for $\mathrm{Y}_{\mathrm{H}_{2} \mathrm{O}_{\omega}}>0$ ), can be utilized for computational purposes in evaluating $Q_{j}$ for use in Eq. (71).

These analytic predictions are tested in Figs. 4 and 5. In the plots, the critical volume percentage of hydrogen

$\% \mathrm{H}_{2}=100 \frac{\mathrm{Y}_{\mathrm{H}_{2 \infty \mathrm{I}}} / \mathrm{W}_{\mathrm{H}_{2}}}{\sum_{\mathrm{i}} \mathrm{Y}_{\mathrm{i}} / \mathrm{W}_{\mathrm{i}}}$

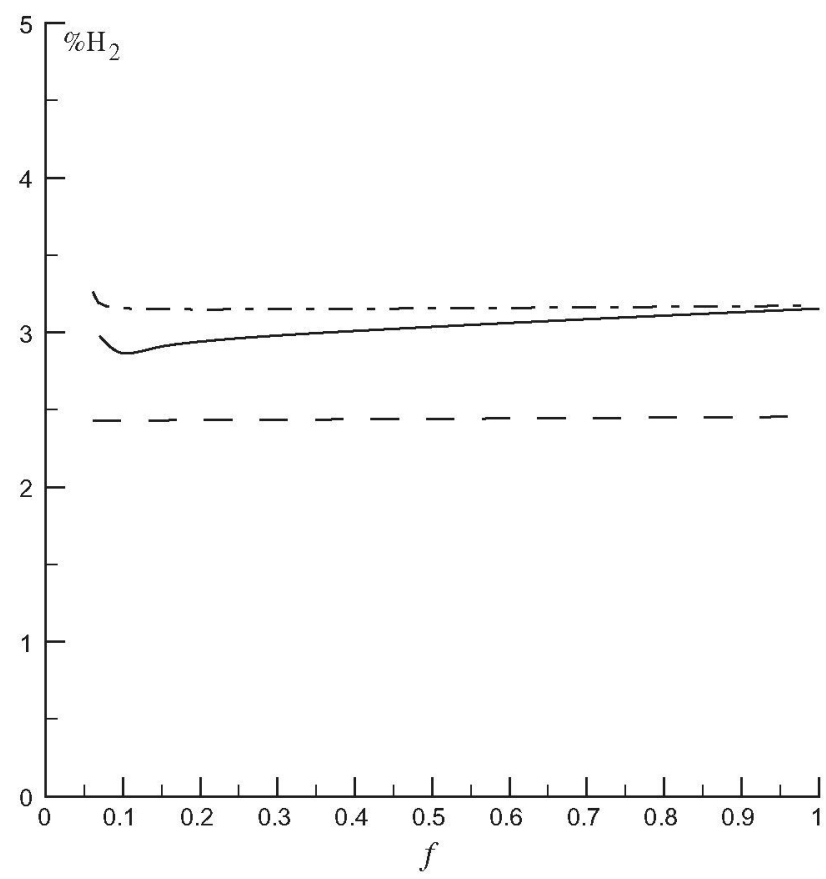

Fig. 4 - Predicted dilution effects; the variation with the enrichment parameter $f=Y_{\mathrm{O}_{2 \infty}} /\left(\mathrm{Y}_{\mathrm{O}_{2 \infty}}+\mathrm{Y}_{\mathrm{N}_{2 \omega}}\right)$ of the limiting volume percentage of hydrogen for flame-ball existence as obtained for a hydrogen-oxygen-nitrogen mixture at $p=1 \mathrm{~atm}$ and $T_{\infty}=300 \mathrm{~K}$ from numerical integrations with detailed chemistry (solid curve), from the leading-order prediction given in Eqs. (31) and (32) (dashed curve), and from the first-order correction given in Eq. (71) (dot-dashed curve).

corresponding to the turning point arising in detailedchemistry computations of steady flame balls is compared with the prediction obtained from the asymptotic analysis at leading order, associated with the value $\mathrm{Y}_{\mathrm{H}_{2 \infty 1}}^{\circ}$, and the corresponding correction, calculated from Eq. (71). The results in Fig. 4 consider a mixture of hydrogen, oxygen, and nitrogen for different values of the enrichment parameter

$f=\frac{Y_{\mathrm{O}_{2 \omega}}}{Y_{\mathrm{O}_{2 \omega}}+\mathrm{Y}_{\mathrm{N}_{2 \infty}}}$

which takes the value $f=0.232$ for a hydrogen-air mixture, and in terms of which $\phi=(8 / f) \mathrm{Y}_{\mathrm{H}_{2 \omega}} /\left(1-\mathrm{Y}_{\mathrm{H}_{2 \omega}}\right)$. The agreement obtained is seen to be very good, with the first-order correction improving considerably the leading-order prediction all the way to small values of $f$, where the system is fuel-rich and the analysis begins to break down. It is seen that the resulting flammability limit is largely independent of the degree of dilution of the oxidizer mixture, with $\% \mathrm{H} \simeq 2.5$ for the leadingorder prediction and $\% \mathrm{H} \simeq 3$ for the corrected prediction and for the numerical results. This independence can be anticipated from the analytical results by noting that variations of $\mathrm{Y}_{\mathrm{O}_{20}}$ only affect the leading-order solution by modifying slightly the mean molecular weight $W$, used in Eq. (33) to determine the third-body concentration, with additional small modifications emerging in the first-order correction through the weak oxygen dependence of the overall reaction rate, entering through the parameters defined in Eqs. (15) and (16). 


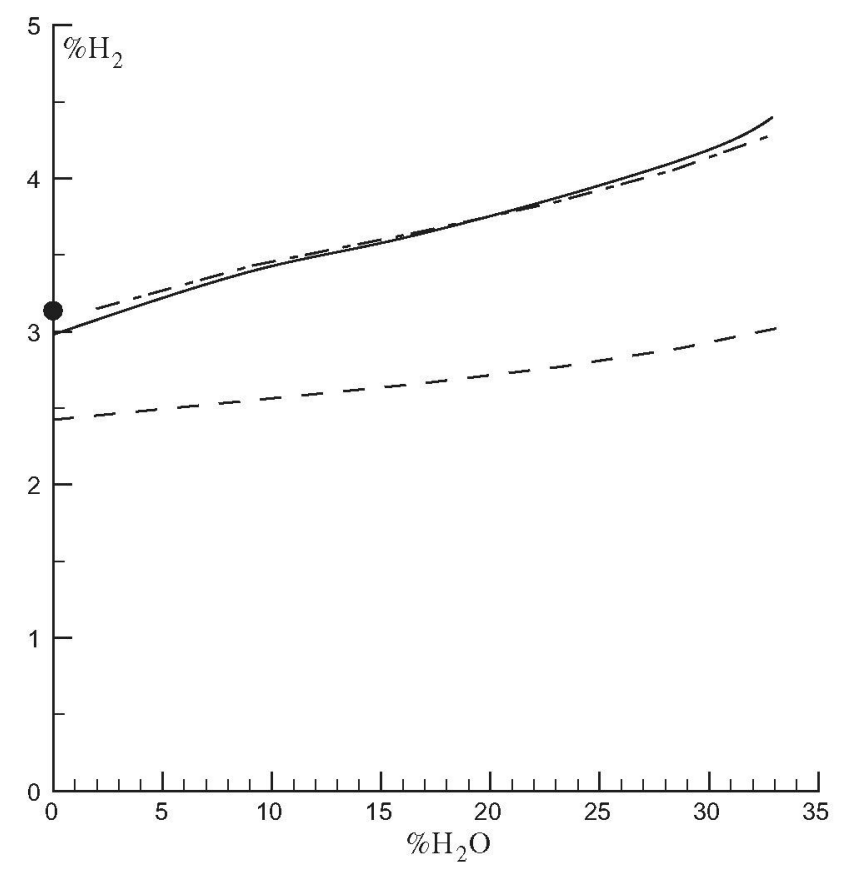

Fig. 5 - Predicted humidity effects; the variation with watervapor content of the limiting percentage of hydrogen for flame-ball existence as obtained for $\mathrm{a}_{2}$-air- $\mathrm{H}_{2} \mathrm{O}$ mixture at $p=1 \mathrm{~atm}$ and $T_{\infty}=300 \mathrm{~K}$ from numerical integrations with detailed chemistry (solid curve), from the leading-order prediction given in Eqs. (31) and (32) (dashed curve), and from the first-order correction Eq. (71) with $Q_{1}$ evaluated from Eq. (73) (dot-dashed curve); the dot indicates the corrected value given in Eq. (71) obtained without ambient water vapor with $Q_{l}$ evaluated from Eq. (72).

A second set of computations, shown in Fig. 5, explores the accuracy of the theoretical results in the presence of ambient humidity, characterized by the volume percentage of water vapor in the ambient mixture

$\% \mathrm{H}_{2} \mathrm{O}=100 \frac{\mathrm{Y}_{\mathrm{H}_{2} \mathrm{O}_{\infty}} / W_{\mathrm{H}_{2} \mathrm{O}}}{\sum_{\mathrm{i}} \mathrm{Y}_{\mathrm{i}} / \mathrm{W}_{\mathrm{i}}}$

In the figure, the first-order correction Eq. (71) is computed for $2<\% \mathrm{H}_{2} \mathrm{O}<35$ with $\mathrm{Q}_{1}$ evaluated from Eq. (73). A dot is used to represent the prediction obtained for $\% \mathrm{H}_{2} \mathrm{O}=0$ with $Q_{l}$ evaluated from Eq. (72). Note that neither of the predictions for $Q_{1}$ given in Eqs. (72) and (73) is strictly valid when the ambient mass fraction $\mathrm{Y}_{\mathrm{H}_{2} \mathrm{O}_{\infty}}$ is comparable with the value, of order ${ }_{\varepsilon} \mathrm{Y}_{\mathrm{H}_{2} \mathrm{O}_{r}}$, that occurs in the far field as a consequence of watervapor diffusion from the flame. The solid and dot-dash curves in Fig. 5 in principle approach the limit of zero watervapor content horizontally, but $\varepsilon$ is sufficiently small that this transition occurs over an extremely small range, too small to be seen in the figure. This transition regime is not investigated further here, because for small values of $\% \mathrm{H}_{2} \mathrm{O}$ both Eqs. (72) and (73) give sufficiently accurate results, that is, the reasoning on which Eq. (72) is based provides an excellent approximation under these conditions.

As can be seen, the resulting value of $\% \mathrm{H}_{2}$ in the $\mathrm{H}_{2}$-air- $\mathrm{H}_{2} \mathrm{O}$ mixture raises with increasing $\mathrm{H}_{2} \mathrm{O}$ content, almost linearly, increasing by about 50\% when the water content reaches 35 percent. This is a consequence of two different effects. On one hand, an increase in the water-vapor concentration increases the crossover temperature, because of the third-body efficiency present in Eq. (33), and therefore increases also the minimum fuel content required to reach $T_{c}$, an effect that is captured at leading order in the radiation-free solution determined from Eqs. (31) and (32). On the other hand, the presence of water vapor also enhances radiation and reduces the flame-ball radius at the turning point, as seen in Fig. 1; this in turn increases the magnitude of the radiation correction $\left(\mathrm{Y}_{\mathrm{H}_{2 \omega}}-\mathrm{Y}_{\mathrm{H}_{2 \omega 0}}^{\circ}\right)$. This second effect is absent at leading order but is described well by the asymptotic analysis accounting for radiation, through the correction term appearing in Eq. (71). Even without ambient water vapor, accounting for radiation through the correction term in Eq. (71) significantly improves predictions (Fig. 4).

\section{Discussion}

Lean near-limit spherical flame balls in hydrogen-oxygendiluent mixtures are predicted here to exhibit a distinct multizone structure. A central spatially uniform hot region is bounded by a thin reaction zone, outside of which heat conduction and molecular diffusion are dominant, over a near-field region extending to roughly ten flame radii and through which the temperature decreases nearly to the ambient temperature. Outside of this region is a far-field region in which radiant heat loss in water-vapor bands and heat conduction are in balance, in a transparent gas. Beyond the far field, at radii on the order of a meter or more, the gas would no longer be optically thin, but that exerts a negligible influence on the flame-ball structure. The far-field region itself is, however, important in that for the near field it produces an effective ambient temperature in excess of the true ambient temperature, thereby modifying fluxes from the near field into the reaction zone. This type of modification reduces the fuel flux, thereby decreasing the heatrelease rate, so that the far-field radiant energy loss detracts from the heat release and thereby renders the flame ball less robust, but it also is essential for stabilizing flame balls, which are known to be unstable in the absence of radiant loss. Analytical solutions have been derived here for the structures of each zone of this interacting multi-zone system.

The focus of the study has been on ultra-lean flammability limits. To accomplish that, in a manner amenable to analytical solutions and matching for the various zones, a distinguished limit was identified, in which an effective large nondimensional overall activation energy $\beta$ for the heat-release rate, given in Eq. (25), scales with small radiation-toconduction rate-ratios, defined in Eqs. (35) and (36), in such a way that the relevant controlling parameters of order unity in the limit can be identified. This scaling serves to produce the two branches of solutions, for flame-ball radius and temperature as functions of the equivalence ratio, found in detailed numerical computations, and it can be used in the future for addressing the stability of the solutions on these two branches, one of which is known to be unstable and the other to have a significant range of stability. The asymptotic development requires the Zeldovich [9] classical radiation-free solution based on Arrhenius kinetics to be modified to incorporate our 
previous overall reaction rate [21,22], derived for lean hydrogen-oxygen mixtures. Even though that structure is entirely unstable, it still provides a reasonable estimate of the lean flammability limit by taking its limit of infinite flame-ball radius, namely equating the flame temperature to the crossover temperature between the branching and recombination rates to give Eq. (31). Improved flammability estimates, however, require development of the next term in the asymptotic expansion including radiation, resulting in the multi-zone structure described above and the two branch solution.

While the flammability limit can be expressed by a simple formula (31) at leading order in the expansion, and the solutions for the structures of the different zones are analytical at the following orders, computation of the flammability limit at the following order entails simultaneous solution of two equations, Eqs. (57) and (64) or Eq. (69), which does not yield an explicit formula for $\mathrm{Q}_{\mathrm{l}}$ Correlations of solutions, given in $\mathrm{Eq}$. (72) or Eq. (73), however, facilitate calculations. The resulting corrections, on the order of ten percent, produce predictions of flammability limits that are in excellent agreement with those calculated by full numerical integrations, noticeably increasing the value of the hydrogen content at the limit above those predicted at leading order. The results show how dilution with $\mathrm{N}_{2}$ does not affect significantly the lean flammability limit and how humidity increases the limiting $\mathrm{H}_{2}$ content through both the high chaperon efficiency of water molecules for recombination and the enhanced radiant energy loss rate.

\section{Conclusions}

This study has predicted lean flammability limits for hydrogen. In dry atmospheres at normal atmospheric pressure and temperature, the limiting volume percentage of hydrogen is predicted to be 3 , a value that remains approximately constant regardless of the relative nitrogen-to-oxygen mixture composition. The presence of water vapor in the atmosphere, however, decreases the range of flammability by increasing influences of radiant energy loss. If, for example, the atmosphere contains 35 percent water vapor by volume, then it is predicted that at least 4.5 percent by volume of $\mathrm{H}_{2}$ would have to be present for the mixture to be flammable. For a hydrogen-air mixture, increasing the pressure to $10 \mathrm{~atm}$ is predicted to increase the critical limiting $\mathrm{H}_{2}$ percentage to 4.2 , and doubling the temperature to $600 \mathrm{~K}$ is predicted to decrease it to approximately 2, as may be deduced from Eqs. (31) and (32), for example. These conclusions, obtained from theoretical analyses of flame-ball structures, would be worth testing in future experiments. Since the predicted limits are the ultimate ones that are possible theoretically, experimental limits are not likely to be quite so broad, except possibly in spacecraft environments, where perturbing effects such as buoyancy are minimized.

\section{Acknowledgments}

This work was supported by the Comunidad de Madrid through project \#P2009/ENE-1597. The first three authors also acknowledge support from the Spanish MCINN through projects \# ENE2008-06515 and CSD2010-00011.

\section{REFERENCES}

[1] Böker D, Brüggemann D. Advancing lean combustion of hydrogen-air mixtures by laser-induced spark ignition. Int $J$ Hydrog Energy 2011;36(22):14759-67.

[2] Wierzba I, Ale BB. Effects of temperature and time of exposure on the flammability limits of hydrogen-air mixtures. Int J Hydrog Energy 1998;23:1197-202.

[3] Swain MR, Filoso PA, Swain MN. Ignition of lean hydrogen-air mixtures. Int J Hydrog Energy 2005;30:1447-55.

[4] Butler MS, Moran CW, Sunderland PB, Axelbaum RL. Limits for hydrogen leaks that can support stable flames. Int J Hydrog Energy 2009;34:5174-82.

[5] Schefer RW, Evans GH, Zhang J, Ruggles AJ, Greif R. Ignitability limits for combustion of unintended hydrogen releases: experimental and theoretical results. Int J Hydrog Energy 2011;36:2426-35.

[6] Lewis B, von Elbe G. Combustion, flames and explosions in gases. 3rd ed. Orlando: Academic Press; 1987.

[7] Shoshin YL, van Oijen JA, Sepman AV, de Goey LPH. Experimental and computational study of the transition to the flame ball regime at normal gravity. Proc Combust Inst 2011;33:1211-8.

[8] Williams FA, Grcar JF. A hypothetical burning-velocity formula for very lean hydrogen-air mixtures. Proc Combust Inst 2009;32:1351-7.

[9] Zelvovich Ya B. Theory of combustion and detonation of gases. Moscow: Izd-vo. Akad. Nauk (Academy of Sciences, USSR); 1944.

[10] Ronney PD. Near-limit flame structures at low Lewis number. Combust Flame 1990;82:1-14.

[11] Ronney PD, Whaling KN, Abbud-Madrid A, Gatto JL, Pisowicz VL. Stationary premixed flames in spherical and cylindrical geometries. AIAA J 1994;32:569-77.

[12] Ronney PD, Wu MS, Weiland KJ, Pearlman HG. Flame ball experiments in space: preliminary results from STS-83. AIAA J 1998;36:1361-8.

[13] Ronney PD. Understanding combustion processes through microgravity research. Proc Combust Inst 1998;27:2485-506.

[14] YaB Zeldovich, Barenblatt CI, Librovich VB, Makhviladze GM. The mathematical theory of combustion and explosions. New York: Consultants Bureau; 1985. pp. 331.

[15] Buckmaster J, Joulin G, Ronney PD. Effects of heat loss on the structure and stability of flame balls. Combust Flame 1990; 79:381-92.

[16] Buckmaster J, Joulin G, Ronney PD. Structure and stability of non-adiabatic flame balls: II. Effects of far-field losses. Combust Flame 1991;84:411-22.

[17] Buckmaster J, Smooke M, Giovangigli V. Analytical and numerical modeling of flame-balls in hydrogen-air mixtures. Combust Flame 1993;94:113-24.

[18] Wu MS, Liu JB, Ronney PD. Numerical simulation of diluent effects in flame ball structure and dynamics. Proc Combust Inst 1998;27:2543-50.

[19] Wu MS, Ronney PD, Colantonio RO, VanZandt DM. Detailed numerical simulation of flame ball structure and dynamics. Combust Flame 1999;116:387-97.

[20] Fernández-Tarrazo E, Sánchez AL, Liñán A, Williams FA. The structure of lean hydrogen-air flame balls. Proc Combust Inst 2011;33:1203-10.

[21] Fernández-Galisteo D, Sánchez AL, Liñán A, Williams FA. One-step reduced kinetics for lean hydrogen-air deflagration. Combust Flame 2009;156:985-96. 
[22] Fernández-Galisteo D, Sánchez AL, Liñán A, Williams FA. The hydrogen-air burning rate near the lean flammability limit. Combust Th Model 2009;13:741-61.

[23] Grcar JF. A new type of steady and stable, laminar, premixed flame in ultra-lean, hydrogenair combustion. Proc Combust Inst 2009;32:1011-8.

[24] Gerlinger W, Schneider K, Bockhorn H. Numerical simulation of three-dimensional instabilities of spherical flame structures. Proc Combust Inst 2000;28:793-9.

[25] Gerlinger W, Schneider K, Fröhlich J, Bockhorn H. Numerical simulations on the stability of spherical flame structures. Combust Flame 2003;132:247-71.

[26] Smooke MD, Giovangigli V. Formulation of the premixed and nonpremixed test problems. In: Smooke MD, editor. Reduced kinetic mechanisms and asymptotic approximations for methane-air flames, Lecture Notes in Physics, vol. 384. , Berlin: Springer-Verlag; 1991. p. 1-28.

[27] Available at: http://maemail.ucsd.edu/combustion/cermech.

[28] Saxena P, Williams FA. Testing a small detailed chemicalkinetic mechanism for the combustion of hydrogen and carbon monoxide. Combust Flame 2006;145:316-23.

[29] Kwon OC, Abid M, Ronney PD, Wu MS, Ju Y. Numerical modeling of flame balls with radiative reabsorption effects. 3rd Joint US Sections Meeting of the Combustion Institute, Chicago, IL, March 21-23, 2003.

[30] RonneyPD. Studies of premixed laminar and turbulent flames at microgravity, NASA Final Report, Grant No. NAG3-2887, 2005.

[31] Minaev S, Kagan L, Joulin G, Sivashinsky G. On self-drifting flame balls. Combust Th Model 2001;5:609-22.

[32] Minaev S, Kagan L, Sivashinsky G. Combust. Selfpropagation of a diffuse combustion spot in premixed gases. Exp Shock Waves 2002;38:9-18.

[33] Fristrom RM, Monchick L. Two simple approximations to the thermal diffusion factor and their applications to flame studies. Combust Flame 1988;71:89-99.

[34] Barlow RS, Karpetis AN, Frank JH, Chen J-Y. Scalar profiles and NO formation in laminar opposed-flow partially premixed methane/air flames. Combust Flame 2001;127:2102-18.

[35] Joulin G. Preferential diffusion and the initiation of lean flames of light fuels. SIAM J Appl Math 1987;47:998-1016.

\section{Nomenclature}

$c_{\mathrm{p}}$ : specific heat at constant pressure of the gas mixture

$\mathrm{c}_{\mathrm{p} i}$ : specific heat at constant pressure of species $i$

$C_{M}$ : effective third-body concentration of reaction $4 f$ given in $E q$. (33)

D: modified fuel diffusivity defined in Eq. (10)

$\mathrm{D}_{\mathrm{i}}$ : diffusivity of species $\mathrm{i}$

f: enrichment parameter defined in Eq. (75)

F: reaction-rate function defined in Eq. (15)

$\mathrm{G}$ : reaction-rate function defined in Eq. (12)

$h_{\mathrm{H}_{2} \mathrm{O}}^{\mathrm{O}}$ : enthalpy of formation of water vapor

$\mathrm{H}$ : reaction-rate function defined in Eq. (13)

$\mathrm{k}_{\mathrm{j}}$ : rate constant of reaction $\mathrm{j}$

$\mathrm{L}_{\mathrm{i}}$ : Lewis number of species $i$

$\mathrm{n}_{\mathrm{j}}$ : exponent for the algebraic temperature dependence $T^{n_{j}}$ of reaction $j$

p: pressure

q: amount of heat released per unit mass of fuel consumed

q: dimensionless heat release defined in Eq. (39)

Q: reduced equivalence ratio defined in Eq. (54)

$Q_{R}$ : radiant heat loss per unit volume

$\tilde{Q}_{R}$ : dimensionless radiant heat loss, as defined in Eq. (40)

$r$ : distance from the flame-ball center

$r_{f}$ : flame-ball radius

$r_{0}$ : characteristic flame-ball radius at extinction, defined in Eq. (34)

$\mathrm{R}_{\mathrm{f}}$ : normalized flame-ball radius defined in Eq. (56)
Ro: universal gas constant

$\mathrm{T}$ : temperature

$T_{a_{j}}$ : activation temperature of reaction $j$

$\mathrm{T}_{\mathrm{c}}$ : crossover temperature

$T_{\mathrm{c}}^{0}$ : value of the crossover temperature determined from Eq. (32)

$\mathrm{T}_{\mathrm{f}}$ : flame-ball temperature

$T_{\infty}$ : ambient temperature

$T_{\infty}^{*}$ : apparent ambient temperature

y: normalized fuel mass fraction defined above Eq. (37)

$\mathrm{y}_{c}$ : normalized fuel mass fraction evaluated at $\theta=1$, as obtained from Eq. (44)

$Y$ : modified fuel mass fraction defined in Eq. (9)

$\mathrm{Y}_{\mathrm{i}}$ : mass fraction of species $\mathrm{i}$

$Y_{H_{2 \infty}:}^{o}$ : leading-oreder prediction for the critical value of the hydrogen mass fraction, as defined in Eq. (31)

$\mathrm{Y}_{\mathrm{H}_{2} \mathrm{O}_{\mathrm{r}}}$ : water-vapor mass fraction at the reaction zone as defined in Eq. (20)

$Y_{\mathrm{O}_{z}}$ : oxygen mass fraction at the reaction zone as defined in Eq. (21)

W: mean molecular weight

$\mathrm{W}_{\mathrm{i}}$ : molecular weight of species $\mathrm{i}$

$\% \mathrm{H}_{2}$ : limiting value of the volume percentage of hydrogen of a flammable mixture as defined in Eq. (74)

$\% \mathrm{H}_{2} \mathrm{O}$ : volume percentage of water vapor in the ambient mixture as defined in Eq. (76)

\section{Greek symbols}

$\alpha$ : reaction-rate function defined in Eq. (14)

$\alpha_{\mathrm{H}_{2}}$ : hydrogen Soret factor

$\beta$ : dimensionless activation energy defined in Eq. (25)

$\gamma$ : exponent for the power-law temperature dependence $\rho D \propto T^{\gamma}$ given in Eq. (24)

$\gamma_{3 \mathrm{~b}}$ : reaction-rate function defined in Eq. (16)

$\Delta$ : radiation parameter defined in Eq. (65)

$\Delta_{W}$ : revised radiation parameter defined in Eq. (70)

$\eta$ : far-field coordinate used in writing Eqs. (59) and (66)

$\varepsilon$ : radiation-to-conduction ratio, as defined in Eq. (35)

$\varepsilon_{\mathrm{W}}$ : radiation-to-conduction ratio in the presence of ambient water vapor, as defined in Eq. (36)

$\kappa_{\mathrm{H}_{2} \mathrm{O}} \mathrm{O}$ : Plank-mean absorption coefficient of water vapor

$\lambda$ : thermal conductivity

$\Lambda$ : dimensionless radiation factor defined in Eq. (61)

$\mu \beta$ : dimensionless activation energy defined in Eq. (26)

$\nu$ : exponent for the power-law temperature dependence $\lambda \propto T^{*}$ given in Eq. (24)

$\omega$ : one-step reaction rate given in Eq. (11)

$\Omega$ : dimensionless fuel-consumption rate defined in Eq. (41)

$\phi$ : equivalence ratio

$\phi_{1}$ : leading-order prediction for the critical value of the minimum equivalence ratio for flame-ball existence

$\phi_{1}^{0}$ : critical minimum equivalence ratio for flame-ball existence

$\rho$ : density

$\sigma:$ Stefan-Boltzmann constant

$\theta$ : dimensionless temperature defined above Eq. (37)

$\tilde{\theta}$ : far-field temperature increment

$\theta_{\infty}^{*}$ : dimensionless value of the apparent ambient temperature

$\Theta_{\mathrm{f}}$ : reduced temperature defined above Eq. (52)

६: dimensionless radial coordinate defined above Eq. (37)

\section{Subscripts}

c: properties evaluated at the crossover temperature

f: properties at $r=r_{f}$

l: properties at the lean flammability limit

$r_{f_{+}}$: properties on the outer side of the reaction sheet

$\xi_{f+}$ : dimensionless properties on the outer side of the reaction sheet

$\xi_{f-}:$ dimensionless properties on the inner side of the reaction sheet

$\infty$ : ambient properties 